\title{
MAGNETIC CAUSES OF SOLAR CORONAL MASS EJECTIONS: DOMINANCE OF THE FREE MAGNETIC ENERGY OVER THE MAGNETIC TWIST ALONE
}

\author{
D. A. Falconer, ${ }^{1}$ R. L. Moore, and G. A. Gary \\ Marshall Space Flight Center, SD50, Space Science Department, Huntsville, AL 35812; \\ david.falconer@msfc.nasa.gov, ron.moore@nasa.gov, allen.gary@nasa.gov. \\ Received 2004 February 2; accepted 2006 February 16
}

\begin{abstract}
We examine the magnetic causes of coronal mass ejections (CMEs) by examining, along with the correlations of active-region magnetic measures with each other, the correlations of these measures with active-region CME productivity observed in time windows of a few days, either centered on or extending forward from the day of the magnetic measurement. The measures are from 36 vector magnetograms of bipolar active regions observed within $\sim 30^{\circ}$ of disk center by the Marshal Space Flight Center (MSFC) vector magnetograph. From each magnetogram, we extract six whole-active-region measures twice, once from the original plane-of-the-sky magnetogram and again after deprojection of the magnetogram to disk center. Three of the measures are alternative measures of the total nonpotentiality of the active region, two are alternative measures of the overall twist in the active-region's magnetic field, and one is a measure of the magnetic size of the active region (the active region's magnetic flux content). From the deprojected magnetograms, we find evidence that (1) magnetic twist and magnetic size are separate but comparably strong causes of active-region CME productivity, and (2) the total free magnetic energy in an active region's magnetic field is a stronger determinant of the active region's CME productivity than is the field's overall twist (or helicity) alone. From comparison of results from the non-deprojected magnetograms with corresponding results from the deprojected magnetograms, we find evidence that (for prediction of active-region CME productivity and for further studies of active-region magnetic size as a cause of CMEs), for active regions within $\sim 30^{\circ}$ of disk center, active-region total nonpotentiality and flux content can be adequately measured from line-ofsight magnetograms, such as from $\mathrm{SOHO}$ MDI.
\end{abstract}

Subject headings: Sun: corona — Sun: coronal mass ejections (CMEs) — Sun: flares — Sun: magnetic fields — Sun: X-rays, gamma rays

\section{INTRODUCTION}

Coronal mass ejections (CMEs) leave the Sun with speeds of up to $\sim 10^{3} \mathrm{~km} \mathrm{~s}^{-1}$ and energies of up to $\sim 10^{32} \mathrm{ergs}$, driving bow shocks that can accelerate protons and other ions to cosmicray energies of $100 \mathrm{MeV}$ or more (Wagner 1984; Kahler 1987; Krimigis 1992; Gosling 1996; Reames 1999). Fast CMEs drive hazardous space weather in the form of intense solar energetic particle events and severe geomagnetic storms (Suess \& Tsurutani 1998; Reames 2001). Development of methods for predicting CMEs is important both for the practical benefit of improved forecasting of damaging space weather and because it is a way to test and improve our physical understanding of these and similar magnetic explosions on the Sun and in other astrophysical settings. For each of a variety of whole-active-region measures from magnetograms, this paper reports on the accuracy of the measure as a predictor of whether an active region will produce a CME during the next few days.

The extent to which CME prediction can be achieved largely depends on how well we can identify solar conditions that make CME production likely. CMEs have been found to erupt from areas exhibiting filaments (Webb et al. 1976), coronal sigmoids and large sunspot area (Canfield et al. 1999), delta sunspots (Zirin \& Liggett 1987), and strongly sheared magnetic fields (Falconer et al. 2002a, 2003). Except for sunspot area, these signatures are all indicators that the magnetic field is highly nonpotential. Based on this characteristic, most theories for the production of

\footnotetext{
${ }^{1}$ Also at Physics Department, University of Alabama at Huntsville, Huntsville, AL 35899.
}

CMEs start with a nonpotential magnetic field (e.g., Sturrock 1989; Moore \& Roumeliotis 1992; Antiochos et al. 1999).

Canfield et al. (1999) found from a study of 107 active regions that active regions that showed sigmoidal coronal structure during disk passage and active regions that had large sunspot area were both statistically significantly correlated with the occurrence of eruptive flares (indicators of CMEs) in the active regions during disk passage. It can be shown from the results presented in Canfield et al. (1999) that (1) sigmoidal active regions had a 65\% CMEcorrelation success rate $(65 \%$ of the active regions either showed sigmoidal structure during disk passage and produced a CME or did not show sigmoidal structure and did not produce a CME), and (2) the classification of active regions as large or small (defined by whether the deprojected sunspot area was greater than or less than 50 millionths of the disk) also had a 65\% CMEcorrelation success rate.

From the above observations, it is reasonable to hypothesize that measurements of active-region magnetograms can be used to predict whether an active region will produce CMEs. In a series of papers (Falconer 2001; Falconer et al. 2002a, 2002b, 2003), we have investigated correlations between certain magnetic measures of bipolar active regions and the CME productivity of these active regions during time windows centered on the day of the magnetogram. Falconer (2001) reported the results of a pilot study of eight vector magnetograms of four bipolar active regions. Two alternative measures of active-region total nonpotentiality were measured: net electric current, $I_{N}$, and length of strongshear main neutral line, $L_{\mathrm{SSM}}$. Two of the four active regions were found to be very nonpotential, while the other two active regions were found to be much more nearly potential. The two highly 
nonpotential active regions produced CMEs during disk passage, while the two roughly potential ones did not. Although the sample was too small to prove our hypothesis, the results were consistent with the hypothesis.

Falconer et al. (2002a) expanded the sample to 17 vector magnetograms of 12 bipolar active regions. In addition to measuring $L_{\mathrm{SSM}}$ and $I_{N}$, we measured the total magnetic flux, $\Phi$, of the active region, a measure of the active region's size. We also normalized the net current by $\Phi$ to get the magnetic twist parameter $\alpha_{\mathrm{IN}}$, a nonpotentiality measure normalized by the size of the active region. Each measure was obtained from a plane-of-thesky magnetogram (i.e., from the observed line-of-sight and transverse field components). We found (1) a statistically significant correlation between each pair of nonpotentiality measures (correlation confidence level $>99 \%$ ); (2) a statistically significant correlation of each nonpotentiality measure with CME productivity during a window of \pm 2 days relative to the day of the magnetogram (correlation confidence level 99\%); (3) that the significance of the correlation between each nonpotentiality measure and CME productivity was higher for the window of \pm 2 days than for windows of either \pm 1 or \pm 4 days. The sample size was not large enough to show a statistically significant correlation between $\Phi$ and CME productivity.

Falconer et al. (2003) introduced a third total-nonpotentiality measure, the length of strong-gradient main neutral line, $L_{\mathrm{SGM}}$, which can be evaluated from a line-of-sight magnetogram alone (without a vector magnetogram). The adjective "strong-gradient" refers to the magnitude of the gradient of the line-of-sight component of the magnetic field $\left(\nabla B_{\|}\right)$at the main neutral line. We expected $L_{\mathrm{SGM}}$ to be an indicator of total nonpotentiality because it is known that on active-region neutral lines across which the gradient in the line-of-sight field is very large, such as in delta sunspots, the magnetic field is nearly always very strongly sheared (e.g., Moore \& Rabin 1985; Zirin \& Liggett 1987; Zirin 1988). Using the sample of Falconer et al. (2002a), we found $L_{\mathrm{SGM}}$ to be statistically significantly correlated with $L_{\mathrm{SSM}}$ (correlation confidence level 99.7\%), and with active-region CME productivity in the \pm 2 day window (correlation confidence level 99.7\%). The success rate of the correlation between $L_{\mathrm{SGM}}$ and $L_{\mathrm{SSM}}$ was $\sim 88 \%$ (15 successes out of 17 trials), and $L_{\mathrm{SGM}}$ and $L_{\mathrm{SSM}}$ each had a $\sim 88 \%$ success rate in its correlation with CME productivity. The importance of this finding is that it opens good-cadence, longrunning sets of synoptic line-of-sight magnetograms, such as from the Solar and Heliospheric Observatory $(\mathrm{SOHO})$ Michelson Doppler Imager (MDI), for use in operational CME forecasting and for study of the dependence of CME productivity on the rate of growth or decay of nonpotentiality.

In this paper, we have added another 19 bipolar active regions to the sample used in Falconer et al. (2002a, 2003), raising the sample size to 36 vector magnetograms of 31 bipolar active regions. These active regions, like the previous ones, each have an obvious main neutral line and have strong transverse field over much $(\gtrsim 20,000 \mathrm{~km})$ of the main neutral line. The results presented in this paper apply only to this restricted class of active region, which are magnetically less complex than active regions that have multiple neutral lines of comparable length rather than having an obvious main neutral line. From each magnetogram we obtain three measures of the total nonpotentiality of the active region, two measures of the overall twist in the active region's magnetic field, and one measure of the size of the active region. Each measure is evaluated from the non-deprojected plane-of-the-sky magnetogram, that is, from the directly observed line-of-sight and transverse field components (as we did in Falconer et al. 2002a, 2003) and is also evaluated from the vector magnetogram deprojected to disk center, that is, from the vertical and horizontal components of the observed vector field (as we did in Falconer 2001). For the deprojected magnetograms, we compare each of the six active region magnetic measures with the CME productivity of the active region in the three time windows $( \pm 1, \pm 2$, and \pm 4 days relative to the day of the magnetogram) that we used in Falconer et al. (2002a). For each window, we evaluate the correlation of each measure with CME productivity and compare the results to the Falconer et al. (2002a) results. For each of the two sets of magnetograms (deprojected or non-deprojected), we use three new forward-looking forecast windows $(0-1,0-2$, and $0-4$ days) to examine the CMEprediction success rates of the six measures. Due to the larger sample, we obtain better estimates of the correlation success rates than could be obtained in Falconer et al. (2002a, 2003).

For each of the three forecast windows, the active-region measures from the deprojected magnetograms perform as follows as CME predictors: (1) the three measures of total nonpotentiality each have a $\sim 75 \%$ CME-prediction success rate, whereas (2) the two measures of overall twist each have only a $\sim 65 \% \mathrm{CME}-$ prediction success rate, and (3) the measure of active region size also has a $\sim 65 \%$ CME-prediction success rate. These results (1) agree with the $\sim 65 \%$ success rate of the Canfield et al. (1999) correlations of eruptive-flare production with active-region sigmoidality and size, and (2) indicate that the total free magnetic energy built up in an active region is a stronger determinant of the active region's CME productivity than is the overall magnetic twist.

For the active-region measures from the non-deprojected magnetograms, the CME-prediction performance differs from that of the measures from the deprojected magnetograms in one important way: whereas the measures of total nonpotentiality and size from non-deprojected magnetograms have the same CME-prediction success rates $(\sim 75 \%$ and $\sim 65 \%)$ as those from the deprojected magnetograms, the twist measures surprisingly have a larger CME-prediction success rate than those from the deprojected magnetograms ( $\sim 75 \%$ instead of $\sim 65 \%$ ). The larger success rate from the non-deprojected magnetograms is apparently a spurious result due to errors in the twist measures from projection effects in the magnetograms. This means that only deprojected magnetograms are acceptable for evaluating the twist measures for use as CME predictors. On the positive side, the relative lack of sensitivity of the total-nonpotentiality and size measures to projection effects means that, for active regions within $30^{\circ}$ of disk center, line-of-sight magnetograms such as from SOHO MDI can be used instead of vector magnetograms to obtain acceptable measures of active-region total nonpotentiality and size for CME prediction.

\section{DATA}

\subsection{Selection of Magnetograms}

The 36 vector magnetograms used in this study are from the Marshall Space Flight Center vector magnetograph (Hagyard et al. 1982; West et al. 2002). The date, NOAA active-region number, longitude, and latitude of each magnetogram are given in Table 1. Each magnetogram is of an active region that fits the following three conditions: The active region (1) is nominally within $\pm 30^{\circ}$ longitude of central meridian (this results in the angle from disk center being less than $30^{\circ}$ for all but seven of the magnetograms, for which the angle is between $30^{\circ}$ and $36^{\circ}$ ); (2) is basically bipolar (has an obvious main neutral line similar to that in Figure 1; and (3) has strong transverse magnetic field on much of the main neutral line (that is, on $\gtrsim 20,000 \mathrm{~km}$ of the main 
TABLE 1

Magnetic Measures and CME Productivity of the Active Regions in our Sample

\begin{tabular}{|c|c|c|c|c|c|c|c|c|c|c|}
\hline No. & $\mathrm{AR}$ & Date & $\begin{array}{l}\text { Longitude, Latitude } \\
\text { (deg) }\end{array}$ & $\begin{array}{c}L_{\mathrm{SSM}} \\
\left(10^{3} \mathrm{~km}\right)\end{array}$ & $\begin{array}{c}L_{\mathrm{SSM}} \\
\left(10^{3} \mathrm{~km}\right)\end{array}$ & $\begin{array}{c}I_{N} \\
\left(10^{11} \mathrm{~A}\right)\end{array}$ & $\begin{array}{c}\alpha_{\mathrm{IN}} \\
\left(10^{-8} \mathrm{~m}^{-1}\right)\end{array}$ & $\begin{array}{c}\alpha_{\mathrm{BC}} \\
\left(10^{-8} \mathrm{~m}^{-1}\right)\end{array}$ & $\begin{array}{c}\Phi \\
\left(10^{21} \mathrm{Mx}\right)\end{array}$ & Day of CME Relative to Day of Magnetogram \\
\hline 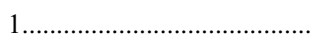 & 6982 & 1991 Dec 26 & W18, S14 & $95 \pm 6^{*}$ & $79 \pm 8^{*}$ & $47 \pm 9^{*}$ & $1.0 \pm 0.1^{*}$ & $4.4 \pm 1.8^{*}$ & $39 \pm 8^{*}$ & $-6,2$ \\
\hline 2 & 7070 & $1992 \mathrm{Feb} 27$ & W8, N7 & $75 \pm 6^{*}$ & $74 \pm 4^{*}$ & $-27 \pm 5^{*}$ & $-0.7 \pm 0.1$ & $0.0 \pm 1.3$ & $32 \pm 6^{*}$ & $-6,-3,0,7$ \\
\hline 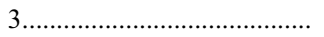 & 7070 & 1992 Feb 28 & W21, N7 & $73 \pm 6^{*}$ & $73 \pm 6^{*}$ & $-25 \pm 5^{*}$ & $-0.6 \pm 0.1$ & $-0.4 \pm 1.2$ & $35 \pm 7^{*}$ & $-7,-4,-1,6$ \\
\hline 4 & 7220 & $1992 \mathrm{Jul} 12$ & $\mathrm{~W} 10, \mathrm{~S} 12$ & $21 \pm 3$ & $24 \pm 12$ & $-2 \pm 1$ & $-0.2 \pm 0.1$ & $0.2 \pm 0.5$ & $13 \pm 2$ & $\ldots$ \\
\hline 5 & 7260 & 1992 Aug 16 & E20, N16 & $51 \pm 12^{*}$ & $3 \pm 9$ & $-29 \pm 5^{*}$ & $-0.6 \pm 0.1$ & $-3.2 \pm 0.8$ & $40 \pm 8^{*}$ & 4 \\
\hline 6 & 7315 & 1992 Oct 19 & E23, N7 & $7 \pm 3$ & $0 \pm 3$ & $9 \pm 2$ & $1.0 \pm 0.1^{*}$ & $-2.6 \pm 0.8$ & $8 \pm 1$ & $\ldots$ \\
\hline 7 & 7315 & 1992 Oct 21 & W2, N5 & $56 \pm 10^{*}$ & $0 \pm 0$ & $-9 \pm 2$ & $-0.5 \pm 0.1$ & $-3.7 \pm 1.0$ & $17 \pm 3$ & $\ldots$ \\
\hline 8 & 7315 & 1992 Oct 23 & W31, S6 & $75 \pm 15^{*}$ & $10 \pm 8$ & $-9 \pm 2$ & $-0.3 \pm 0.0$ & $-2.7 \pm 0.9$ & $27 \pm 5^{*}$ & $\ldots$ \\
\hline 9 & 7999 & 1996 Nov 27 & W17, S4 & $32 \pm 9$ & $0 \pm 3$ & $-13 \pm 3^{*}$ & $-0.5 \pm 0.1$ & $-1.8 \pm 1.3$ & $21 \pm 4^{*}$ & 3 \\
\hline $10 \ldots \ldots \ldots$ & 8083 & 1997 Sep 07 & E6, S27 & $0 \pm 0$ & $0 \pm 0$ & $-11 \pm 2$ & $-1.0 \pm 0.1^{*}$ & $-2.5 \pm 1.0$ & $9 \pm 1$ & $\ldots$ \\
\hline 11 & 8100 & 1997 Oct 31 & E21, S19 & $25 \pm 3$ & $22 \pm 5$ & $-4 \pm 1$ & $-0.4 \pm 0.1$ & $-4.0 \pm 2.0^{*}$ & $8 \pm 1$ & $3,5,6$ \\
\hline 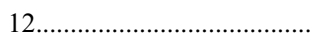 & 8100 & 1997 Nov 03 & W21, S19 & $105 \pm 6^{*}$ & $35 \pm 19^{*}$ & $-20 \pm 4^{*}$ & $-1.0 \pm 0.1^{*}$ & $-5.7 \pm 3.1^{*}$ & $16 \pm 3$ & $0,2,3$ \\
\hline 13 & 8108 & 1997 Nov 18 & E20, N21 & $0 \pm 0$ & $0 \pm 0$ & $4 \pm 1$ & $0.2 \pm 0.0$ & $0.5 \pm 0.6$ & $15 \pm 3$ & $-4,-3,1$ \\
\hline 14 & 8323 & 1998 Sep 02 & E2, S23 & $139 \pm 35^{*}$ & $31 \pm 28^{*}$ & $-2 \pm 1$ & $-0.1 \pm 0.0$ & $-0.7 \pm 0.7$ & $32 \pm 6^{*}$ & $\ldots$ \\
\hline 15 & 9026 & 2000 Jun 06 & E14, N21 & $129 \pm 17^{*}$ & $66 \pm 9^{*}$ & $-25 \pm 5^{*}$ & $-1.6 \pm 0.2^{*}$ & $-9.6 \pm 3.7^{*}$ & $13 \pm 2$ & $-3,0,1,4$ \\
\hline 16 & 9077 & $2000 \mathrm{Jul} 14$ & W6, N17 & $161 \pm 26^{*}$ & $96 \pm 19^{*}$ & $-38 \pm 8^{*}$ & $-1.1 \pm 0.1^{*}$ & $-8.5 \pm 5.9^{*}$ & $29 \pm 5^{*}$ & $-4,-3,-2,0$ \\
\hline 17 & 9077 & $2000 \mathrm{Jul} 16$ & W30, N18 & $287 \pm 30^{*}$ & $125 \pm 34^{*}$ & $-22 \pm 4^{*}$ & $-0.8 \pm 0.1^{*}$ & $-8.7 \pm 8.1^{*}$ & $23 \pm 4^{*}$ & $-6,-5,-4,-2$ \\
\hline 18 & 9173 & 2000 Sep 29 & E16, S12 & $5 \pm 4$ & $10 \pm 5$ & $-7 \pm 1$ & $-0.5 \pm 0.1$ & $-2.7 \pm 0.5$ & $13 \pm 2$ & $\ldots$ \\
\hline 19 & 9178 & 2000 Oct 03 & W0, S22 & $2 \pm 1$ & $15 \pm 4$ & $-2 \pm 0$ & $-0.2 \pm 0.0$ & $-2.3 \pm 0.4$ & $10 \pm 2$ & $-5,-2,1$ \\
\hline 20 & 9227 & 2000 Nov 11 & $\mathrm{E} 15, \mathrm{~S} 13$ & $0 \pm 1$ & $9 \pm 2$ & $-7 \pm 1$ & $-0.9 \pm 0.1^{*}$ & $-4.2 \pm 0.9^{*}$ & $7 \pm 1$ & $\ldots$ \\
\hline 21 & 9231 & 2000 Nov 19 & W3, S24 & $49 \pm 5^{*}$ & $22 \pm 8$ & $-6 \pm 1$ & $-0.6 \pm 0.1$ & $-6.1 \pm 1.6^{*}$ & $9 \pm 1$ & -5 \\
\hline 22 & 9240 & 2000 Nov 28 & $0 \mathrm{~W}, \mathrm{~N} 8$ & $44 \pm 5$ & $0 \pm 1$ & $-25 \pm 5^{*}$ & $-1.2 \pm 0.1^{*}$ & $-5.0 \pm 0.7^{*}$ & $17 \pm 3$ & -3 \\
\hline 23 & 9254 & 2000 Dec 08 & E1, N10 & $29 \pm 2$ & $21 \pm 6$ & $-13 \pm 2^{*}$ & $-2.2 \pm 0.2^{*}$ & $-4.1 \pm 0.9^{*}$ & $5 \pm 1$ & $\ldots$ \\
\hline 24 & 9267 & 2000 Dec 15 & W10, N7 & $0 \pm 2$ & $0 \pm 0$ & $1 \pm 0$ & $0.1 \pm 0.0$ & $0.4 \pm 0.2$ & $9 \pm 1$ & $\ldots$ \\
\hline 25 & 9289 & 2001 Jan 04 & W33, S7 & $0 \pm 1$ & $0 \pm 1$ & $-1 \pm 0$ & $0.0 \pm 0.0$ & $0.5 \pm 0.1$ & $20 \pm 4^{*}$ & $\ldots$ \\
\hline 26 & 9330 & $2001 \mathrm{Feb} 03$ & E22, N26 & $15 \pm 6$ & $0 \pm 1$ & $0 \pm 0$ & $0.0 \pm 0.0$ & $2.0 \pm 0.6$ & $17 \pm 3$ & $\ldots$ \\
\hline 27 & 9370 & 2001 Mar 09 & W20, N10 & $2 \pm 1$ & $40 \pm 7^{*}$ & $-6 \pm 1$ & $-0.5 \pm 0.1$ & $-1.8 \pm 0.4$ & $11 \pm 2$ & -1 \\
\hline 28 & 9373 & 2001 Mar 13 & $\mathrm{E} 25, \mathrm{~S} 8$ & $5 \pm 5$ & $0 \pm 0$ & $5 \pm 1$ & $0.2 \pm 0.0$ & $0.9 \pm 0.2$ & $20 \pm 4$ & $\ldots$ \\
\hline 29 & 9390 & 2001 Mar 26 & W2, N14 & $9 \pm 7$ & $29 \pm 8^{*}$ & $1 \pm 0$ & $0.1 \pm 0.0$ & $0.5 \pm 0.3$ & $10 \pm 2$ & -2 \\
\hline 30... & 9396 & 2001 Mar 26 & W25, S6 & $19 \pm 3$ & $1 \pm 6$ & $7 \pm 1$ & $0.8 \pm 0.1^{*}$ & $2.7 \pm 0.5$ & $8 \pm 1$ & $\ldots$ \\
\hline $31 \ldots$ & 9445 & 2001 May 04 & $\mathrm{E} 1, \mathrm{~N} 25$ & $18 \pm 10$ & $56 \pm 8^{*}$ & $0 \pm 0$ & $0.0 \pm 0.0$ & $1.3 \pm 0.4$ & $23 \pm 4^{*}$ & 6 \\
\hline 32 & 9463 & 2001 May 22 & $\mathrm{E} 22, \mathrm{~N} 12$ & $2 \pm 3$ & $0 \pm 1$ & $6 \pm 1$ & $0.3 \pm 0.0$ & $2.4 \pm 0.4$ & $17 \pm 3$ & $\ldots$ \\
\hline 33 & 9502 & 2001 Jun 16 & $\mathrm{E} 23, \mathrm{~S} 25$ & $29 \pm 9$ & $134 \pm 38^{*}$ & $-3 \pm 0$ & $-0.3 \pm 0.0$ & $-4.0 \pm 1.7^{*}$ & $10 \pm 2$ & $-5,-3,-2,-1,6$ \\
\hline $34 \ldots \ldots$ & 9504 & 2001 Jun 19 & $\mathrm{E} 2, \mathrm{~N} 8$ & $9 \pm 4$ & $5 \pm 2$ & $1 \pm 0$ & $0.1 \pm 0.0$ & $-0.1 \pm 0.2$ & $24 \pm 4^{*}$ & $\ldots$ \\
\hline 35 & 9506 & 2001 Jun 19 & E25, N17 & $49 \pm 3$ & $3 \pm 2$ & $17 \pm 3^{*}$ & $4.1 \pm 0.4^{*}$ & $6.5 \pm 1.5^{*}$ & $3 \pm 0$ & $\ldots$ \\
\hline $36 \ldots \ldots \ldots$ & 9543 & $2001 \mathrm{Jul} 19$ & $\mathrm{E} 25, \mathrm{~S} 23$ & $6 \pm 2$ & $6 \pm 9$ & $-6 \pm 1$ & $-0.3 \pm 0.0$ & $-1.2 \pm 0.3$ & $18 \pm 3$ & $\ldots$ \\
\hline Adopted threshold............ & $\ldots$ & $\ldots$ & $\ldots$ & 49 & 29 & 13 & 0.8 & 4.0 & 20 & $\ldots$ \\
\hline
\end{tabular}

NoтE.-Each entry marked by an asterisk is greater than or equal to the adopted threshold value. 


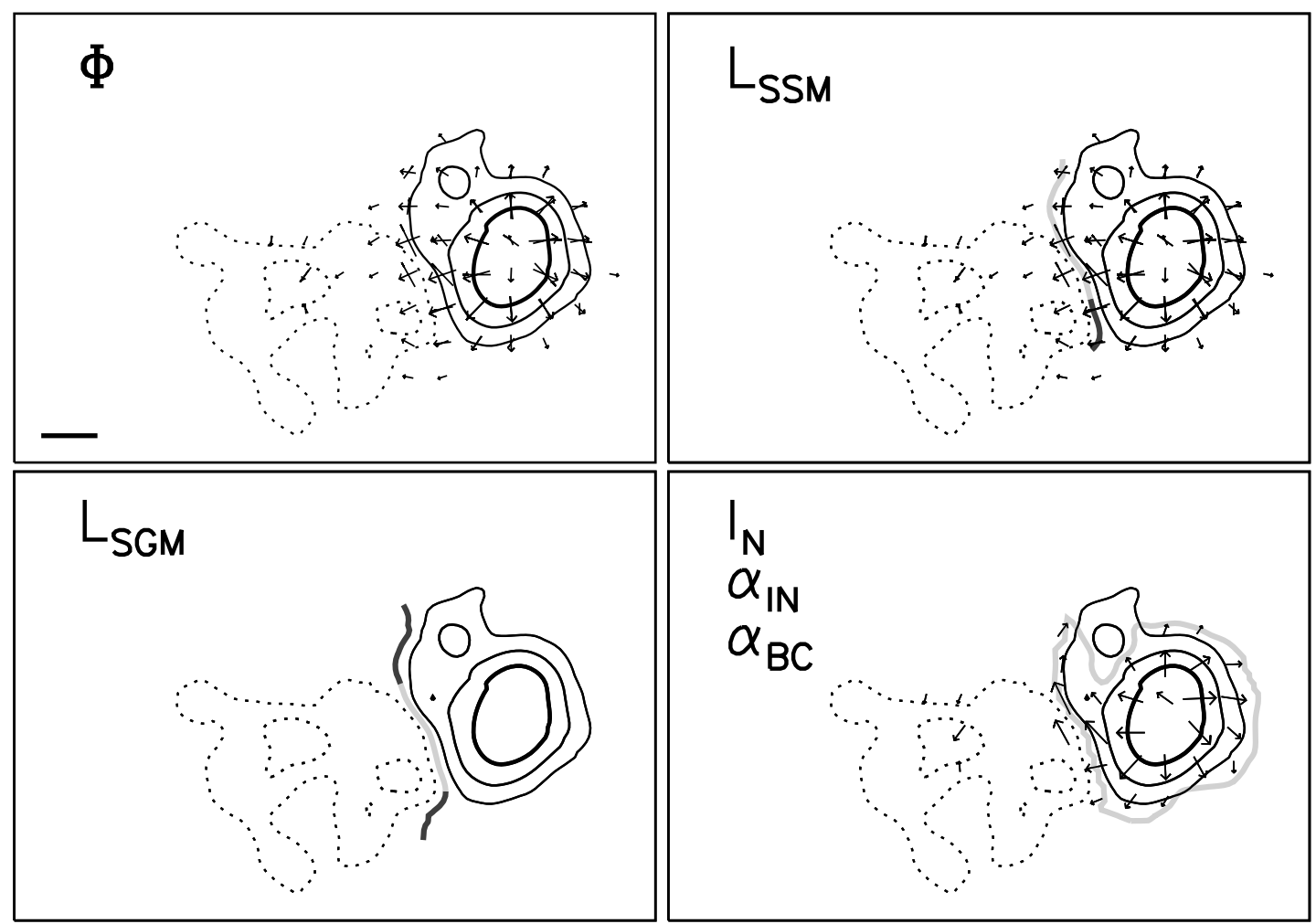

Fig. 1.-Display of the elements of our magnetic measures in an example active region. A subfield of view $(115 \times 80 \mathrm{Mm})$ of the deprojected MSFC vector magnetogram of AR 9254 from 2000 December 8 is shown. The contours in each panel map the strength and polarity of the vertical field component $B_{v}$, with thickness representing field strength $(100,250$, and $500 \mathrm{G})$ and solid (dotted) contours indicating positive (negative) polarity. The arrows and dashes map the strength and direction of the horizontal fields; the shortest are for $150 \mathrm{G}$, the longest are for $\geq 500 \mathrm{G}$, and only 1 in 49 pixels are plotted for clarity. Top left: The potential (arrows) and observed (dashes) horizontal fields are shown. A large shear angle (the acute angle between the potential and observed horizontal field directions) indicates that the magnetic field is strongly sheared, as does the observed horizontal field near the neutral line being nearly parallel to the neutral line. The total magnetic flux $(\Phi)$ is the total absolute magnetic flux from pixels with $\left|B_{v}\right|>100 \mathrm{G}$. The bar in the bottom left corner is 10,000 km long. Top right: The arrows and dashes are the same as in the top left panel. The thick gray curve is the extent of the main neutral line on which the observed horizontal field is greater than $150 \mathrm{G}$. The shading indicates whether the shear angle is larger (light gray) or smaller (dark gray) than $45^{\circ} . L_{\mathrm{SSM}}$ is the length of the light gray interval. Bottom left: The thick gray curve is the part of the main neutral line on which the potential horizontal field is greater than $150 \mathrm{G}$. The shading indicates whether $\left|\nabla B_{v}\right|$ is larger (light gray) or smaller (dark gray) than $50 \mathrm{G} \mathrm{Mm}^{-1}$. The length of the light gray interval is $L_{\mathrm{SGM}}$. Bottom right: The directionalized observed horizontal field (arrows) is shown. The net current $\left(I_{N}\right)$ is evaluated by applying Ampere's law to the integration contour ( gray) to find the net vertical current. The parameter $\alpha_{\mathrm{IN}}$ is obtained from $I_{N}$ and $\Phi$. The twist parameter $\alpha_{\mathrm{BC}}$ is the $\alpha$ of the constant- $\alpha$ horizontal field that best matches the direction of the observed horizontal field weighted by the strength of the observed horizontal field.

neutral line, the transverse field strength is greater than $150 \mathrm{G}$ [this threshold is twice the $75 \mathrm{G}$ noise level of the transverse field in our magnetograms]). The first condition limits deprojection errors, the second limits our sample to active regions of rather simple magnetic structure, and the third is for accurate measurement of quantities obtained from the transverse field.

The sample consists of 17 magnetograms from 12 active regions, the sample used in Falconer et al. (2002a, 2003), plus a magnetogram from each of 19 new active regions. The 19 new magnetograms sample every active region observed by the MSFC vector magnetograph from 2000 September 15 through 2001 July 19 , for which we have at least one magnetogram that fits the three conditions. The magnetogram analyzed for each new active region was typically the one closest to disk center, although seeing quality and how well the active region fit the second and third conditions were taken into account in selecting the magnetogram. In all cases the magnetogram was selected before we determined whether the active region produced any CMEs during disk passage.

\subsection{Magnetogram Calibration, Directionalization, and Deprojection}

Vector magnetograms consist of line-of-sight and transverse magnetic field maps. Measurement of the transverse field in ad- dition to measurement of the line-of-sight field differentiates a vector magnetograph (e.g., MSFC) from a line-of-sight magnetograph (e.g., $S O H O \mathrm{MDI}$ ). The line-of-sight field, $B_{\|}$, is measured from the circular polarization of light in a magnetically sensitive spectral line (Zeeman effect). The MSFC filter vector magnetograph uses the Fe I $5250.2 \AA$ line. In our MSFC vector magnetograms, the strength of the line-of-sight magnetic field is calibrated against co-aligned Kitt Peak magnetograms using a procedure similar to Berger \& Lites (2003). When a Kitt Peak magnetogram is not available, an MDI magnetogram is used instead. We have found from the days that we have both Kitt Peak and MDI that the MDI reported field strength ranges from $66 \%$ to $88 \%$ weaker, with an average of $80 \%$ weaker (Berger \& Lites 2003 found the field strength from MDI to be $64 \%$ weaker than that from ASP). Thus, when there is no Kitt Peak magnetogram we calibrate against the MDI line-of-sight field multiplied by a factor of 1.25. A potential transverse magnetic field is computed from the observed line-of-sight magnetic field (e.g., Falconer 1997). This potential magnetic field is used for measuring the shear angle in the non-deprojected magnetogram.

The transverse magnetic field is measured from the linear polarization of light in the spectral line. There is a $180^{\circ}$ ambiguity in the measured transverse field; that is, only the strength and the line of the transverse field vector, but not its direction along 
the line, are obtained directly from the linear polarization. We resolve the $180^{\circ}$ ambiguity as follows. First we compare the observed to the potential transverse field and pick the direction that makes the shear angle (the angle between the observed and potential transverse field) acute. For most pixels this is the correct direction, but in areas of strong shear the shear angle for some pixels can be larger than $90^{\circ}$. To identify cases in which this occurs and correct these pixels, we discern the sense (handedness) of the magnetic shear in these strong-shear areas from Yohkoh SXT coronal images (Falconer 2000). We then choose the transverse-field direction that gives that sense of shear to all pixels that have acute-angle shear greater than $55^{\circ}$.

There are three primary sources of uncertainty in our magnetograms (see Falconer 2001; Falconer et al. 2002a). These are (1) uncertainty in the calibration of magnetic field strength, (2) atmospheric seeing, and (3) limited polarization sensitivity, which produces a $1 \sigma$ uncertainty of $\sim 50 \mathrm{G}$ in the line-of-sight field and a $1 \sigma$ uncertainty of $\sim 75 \mathrm{G}$ in the transverse field. The uncertainty from calibration and seeing is estimated to be $\sim 20 \%$ (Falconer 2001; Falconer et al. 2002a). The line-of-sight and transverse field uncertainties due to polarization sensitivity are determined for each magnetogram as follows from a co-aligned magnetogram of the active region from a full-disk magnetogram from Kitt Peak or MDI. For the uncertainty in the line-of-sight field $\left(\sigma_{\|}^{\mathrm{MSFC}}\right)$ we first compute

$$
\sigma_{\|}=\left[\frac{1}{N} \Sigma\left(B_{\|}^{\mathrm{MSFC}}-B_{\|}^{\mathrm{FD}}\right)^{2}\right]^{0.5}
$$

where $B_{\|}^{\mathrm{MSFC}}$ is the line-of-sight field from the MSFC magnetogram, $B_{\|}^{\mathrm{FD}}$ is the line-of-sight field from the full-disk magnetogram, $N$ is the number of pixels being summed over, and $\sigma_{\|}$is the total uncertainty, which includes uncertainty due to coalignment errors and evolutionary changes between the two magnetograms, uncertainty in the full disk magnetogram $\left(\sigma_{\|}^{\mathrm{FD}}\right)$, and the actual uncertainty in $B_{\|}^{\mathrm{MSFC}}$ due to polarization noise $\left(\sigma_{\|}^{\mathrm{MSFC}}\right)$. To conservatively estimate $\sigma_{\|}^{\mathrm{MSFC}}$, we neglect the coalignment errors and evolution and assign an $8 \mathrm{G}$ uncertainty for $\sigma_{\mathrm{FD}}$ (Jones et al. 1992; Scherrer et al. 1995) and estimate the uncertainty of $\sigma_{\|}^{\mathrm{MSFC}}$ from

$$
\sigma_{\|}^{\mathrm{MSFC}}=\left[\sigma_{\|}^{2}-\left(\sigma_{\|}^{\mathrm{FD}}\right)^{2}\right]^{0.5} .
$$

To estimate the uncertainty in the transverse field $\left(\sigma_{\perp}\right)$ we use the method of Gary et al. (1987). We first identify those quietSun pixels that should have negligible real transverse field (pixels with line-of-sight field of less than $100 \mathrm{G}$ and potential transverse field of less than $25 \mathrm{G}$ ). In these pixels any observed transverse field is dominated by noise, and so we set

$$
\sigma_{\perp}=\left[\Sigma\left(B_{\perp}^{2}\right) / N\right]^{0.5} .
$$

The magnitude and uncertainty of the transverse field are used to find the uncertainty in the angle of the transverse field, and in turn the uncertainty in the shear angle of the transverse field.

Each of our magnetic measures is a measure of a well-defined physical aspect of the active region's magnetic field when the measure is evaluated from the vertical and horizontal field components of the vector magnetogram. For an active region not at disk center, a measure evaluated from the line-of-sight and transverse field components of the observed vector magnetogram is an approximation of the physical measure that would have been obtained if the active region were at disk center. The accuracy of the approximation depends on the magnetic configuration of the active region and worsens with distance of the active region from disk center.

To evaluate our magnetic measures from the vertical and horizontal components of the vector magnetic field, we effectively deproject the observed vector magnetogram to disk center. That is, we transform the observed line-of-sight and transverse field components to the vertical and horizontal components using the equations of Gary \& Hagyard (1990). The transformation requires the observed vector magnetogram to have been directionalized, that is, to have had its $180^{\circ}$ ambiguity resolved. We use the directionalization method described above. The uncertainty $\left(\sigma_{\|}\right)$in the line-of-sight field and the uncertainty $\left(\sigma_{\perp}\right)$ in the transverse field are transformed to the uncertainties ( $\sigma_{v}$ and $\left.\sigma_{h}\right)$ in the vertical and horizontal fields. We also correct the span of the deprojected magnetogram for the projection foreshortening in the observed magnetogram. From the vertical field component of the deprojected magnetogram, a second potential magnetic field is computed. From the horizontal component of this potential field and the observed horizontal field, we then obtain the shear angle of the horizontal field in each pixel.

\subsection{Magnetogram Measures}

We extract six whole-active-region magnetic measures from each deprojected vector magnetogram and also from each nondeprojected vector magnetogram. In this section we describe how each of these measures is obtained from a deprojected magnetogram. The corresponding measure from the non-deprojected magnetogram is obtained in the same way from the line-of-sight and transverse components of the non-deprojected magnetogram instead of from the vertical and horizontal components of the deprojected magnetogram. To indicate that a measure has been obtained from the non-deprojected magnetogram instead of from a deprojected magnetogram, we add the superscript prefix " $n$ " to the symbol for that measure (e.g., ${ }^{n} L_{\mathrm{SSM}}$ ).

Of the six measures, one is a measure of the active region's size, the total magnetic flux, $\Phi$ (Fisher et al. 1998). Two are measures of overall twist in the active region's magnetic field; these are sizenormalized measures of the active region's nonpotentiality - that is, they have no intrinsic dependence on the size of the active region. The two magnetic-twist measures are the net current alpha, $\alpha_{\mathrm{IN}}$, and the best constant alpha $\alpha_{\mathrm{BC}}$. The other three measures are measures of the total nonpotentiality of the active region; each depends intrinsically on the size of the active region, as well as on the overall twist or shear in the active region's magnetic field. The three total-nonpotentiality measures are the net current, $I_{N}$, the length of strong-field strong-shear main neutral line, $L_{\mathrm{SSM}}$, and the length of strong-field strong-gradient main neutral line, $L_{\mathrm{SGM}}$.

Our six magnetic measures are specified and measured as follows.

1. The total magnetic flux $\Phi$ is the sum of unsigned vertical magnetic flux for all pixels that have vertical magnetic field strength greater than $100 \mathrm{G}$.

2. The lengths $L_{\mathrm{SSM}}$ and $L_{\mathrm{SGM}}$ are determined by first identifying the main neutral line (the $0 \mathrm{G}$ contour that divides the main bipole of the active region). The main neutral line is then divided into many intervals of roughly 1 pixel in length. For each interval, the shear angle, the horizontal gradient of the vertical magnetic field, the interval length, and the magnitudes of the observed and potential horizontal fields are evaluated at the midpoint of the interval. The measure $L_{\mathrm{SSM}}$ is the total length of all intervals for which the observed horizontal field is greater 
than $150 \mathrm{G}$ and the shear angle is greater than $45^{\circ}$ (Fig. 1, top right panel). The measure $L_{\mathrm{SGM}}$ is the total length of all intervals that have the potential horizontal field greater than $150 \mathrm{G}$ and the gradient of the vertical field greater than $50 \mathrm{G} \mathrm{Mm}^{-1}$ (Fig. 1, bottom left panel). Falconer et al. (2003) showed that the correlation of $L_{\mathrm{SGM}}$ with either $L_{\mathrm{SSM}}$ or CME productivity is not sensitive to the choice of gradient threshold: essentially the same results were found for thresholds ranging from 25 to $100 \mathrm{G} \mathrm{Mm}^{-1}$.

3. The measure $\alpha_{\mathrm{BC}}$ is the value of the force-free field parameter that minimizes the differences in direction between the observed and the constant-alpha horizontal field vectors (Pevtsov et al. 1995). It is found by calculating force-free horizontal magnetic fields from the vertical magnetic field for many values of $\alpha$. Then for each value of $\alpha$, we find, over all pixels for which both the observed and the potential horizontal fields are greater than $150 \mathrm{G}$, the average difference between the direction of the observed and the constant-alpha horizontal field vectors weighted by the observed horizontal field strength. Then we plot this weighted-average difference angle versus $\alpha$ and by curve-fitting find the find the best constant $\alpha$, the value of $\alpha$ that gives the minimum weighted average of the difference angle. In strongly nonpotential active regions, the absolute value of $\alpha_{\mathrm{BC}}$ is usually less than that of the $\alpha$ that best fits the strongly sheared horizontal field along the main neutral line but greater than that of the $\alpha$ that best fits the weakly sheared fields far from the main neutral line.

4. The net vertical current $I_{N}$ is the net vertical electric current in one of the two polarity domains of the bipolar active region. There are two ways of evaluating $I_{N}$. One is by evaluating the area integral of the current density map $(\nabla \times \boldsymbol{B})$, and the other is by evaluating a contour integral using Ampere's law $\left(I_{N}=\right.$ $\left.\int \boldsymbol{B}_{h} \cdot d \boldsymbol{l}\right)$, where $\boldsymbol{B}_{h}$ is the horizontal magnetic field vector. In either method, only the net vertical current is measured. We use the contour integral method (Falconer 2001). The integration contour is the contour of the area of one polarity in which the 3 pixel smoothed vertical field is greater than or equal to $33 \mathrm{G}$ $(2 \sigma)$, and the single-pixel horizontal field strength is greater than or equal to $150 \mathrm{G}(2 \sigma)$. The first condition extends the contour toward the edges of the active region to capture as much of the current as possible, while the second one ensures that the horizontal field in the integral is always at least $2 \sigma$ to avoid excessive noise. The more compact of the two magnetic polarity domains of the bipolar active region is chosen for the integration contour. If both polarity domains are of similar compactness, the leading (westward) one is chosen. The sign of the net current is positive (negative) if the current flows up (down) in the positive domain. The net current should not be confused with the total current. We do not attempt to measure the total current for two reasons: (a) our magnetograms do not resolve the smallest current elements, and so underestimate the total current; (b) noise in the direction of the horizontal field or errors in ambiguity resolution tend to overestimate the total current but affects the net current only if the error is on the integration contour (in our magnetograms these errors are greatest in the middle of sunspot umbras, and these are avoided by the integration contour).

5. The magnetic twist parameter $\alpha_{\mathrm{IN}}$ is defined by $\alpha_{\mathrm{IN}}=$ $\mu I_{N} / \Phi$ (Falconer et al. 2002a).

For strongly nonpotential active regions, the two $\alpha$ parameters and $I_{N}$ have the same sign (sense of twist). The few active regions in Table 1 that have mixed signs are all nearly potential with some measures having values less than their uncertainties. For CME prediction we use only the absolute values of $I_{N}, \alpha_{\mathrm{IN}}$, and $\alpha_{\mathrm{BC}}$.

The uncertainty in each of our magnetic measures is estimated as follows. The uncertainty in $\Phi$ is a combination of polarization noise in the vertical field $\left(\sigma_{v}\right)$ and the $20 \%$ uncertainty from calibration and seeing, which strongly dominates the polarization uncertainty. The uncertainty in the two main-neutral-line length measures is determined by evaluating for each interval of the main neutral line the uncertainties in the shear angle, in the horizontal gradient of the vertical magnetic field, and in the strengths of the observed and potential horizontal fields. These uncertainties affect whether an interval is classified as either strong or weak shear (or gradient), and thus whether it contributes to $L_{\mathrm{SSM}}$ (or $L_{\mathrm{SSM}}$ ). A Monte Carlo technique is used to estimate the total uncertainty in these main-neutral-line length measures. The uncertainty in $\alpha_{\mathrm{BC}}$ is determined from the uncertainty in the curve fit that gives $\alpha_{\mathrm{BC}}$. The uncertainty of $I_{N}$ arises from the $20 \%$ calibration/seeing uncertainty as well as the uncertainty in the strength and direction of the horizontal field along each segment of the integration contour. The uncertainty in $\alpha_{\mathrm{IN}}$ is determined by propagation of errors from the two components of $\alpha_{\mathrm{IN}}, I_{N}$ and $\Phi$. The $20 \%$ calibration uncertainty is reduced for $\alpha_{\mathrm{IN}}$ since for any magnetogram, $I_{N}$ and $\Phi$ are equally affected; we estimate that a $10 \%$ uncertainty in $\alpha_{\mathrm{IN}}$ remains from calibration of the transverse field from the line-ofsight field.

\subsection{Determination of CME Productivity}

To determine the CME productivity of an active region during its disk passage, we identify all ejective flares that occur on the main neutral line. We use Yohkoh SXT and GOES observations, and when possible confirm the production of a CME using $\mathrm{SOHO}$ LASCO movies. See Falconer et al. (2002a) for details of the procedure. For each of our active-region magnetograms, the days on which the active regions produced a $\mathrm{CME}$, relative to the day of the magnetogram, are given in the last column of Table 1.

An active region is considered to have been CME-productive during a window of time if it produced at least one CME in that window. We use $\pm N$ day windows (a window centered on the day of the magnetogram) and $0-N$ day windows (forward-looking from the day of the magnetogram). The best value of $N$ (the value giving the highest correlation of a measure with the CME productivity) is a trade-off between two factors: (1) Even strongly CME productive active regions usually produce less than one CME per day (Table 1 of this paper and the discussion in $\S 3.2$ of Falconer et al. 2002a) and (2) active regions can evolve in a few days from weakly to strongly nonpotential (e.g., AR 8100 in Table 1), or from strongly to weakly nonpotential. To cover the best value of $N$, we use $N$ s of 1, 2, and 4, as we did in Falconer et al. (2002a). The $\pm N$ day windows are used in this paper both for comparison to Falconer et al. (2002a, 2003) and to further examine the dependence of the strengths of the correlation on window size. The $0-N$ day windows are used to examine the accuracy of each measure as a predictor of CMEs.

\subsection{Thresholds for CME Expectation}

To assess the degree to which strongly nonpotential or large active regions are more likely to produce CMEs than weakly nonpotential or small active regions, we adopt a threshold for each measure at and above which the measure is large enough that the active region is expected to be CME-productive, and below which the active region is expected to not be CME-productive. Since this is done for each measure, an active region might be expected to be CME-productive from some measures and be expected to not be CME-productive from other measures. That some active regions have mixed expectations allows us to examine through statistical evaluation whether some measures are more accurate than others as predictors of CME productivity (see the Appendix). 

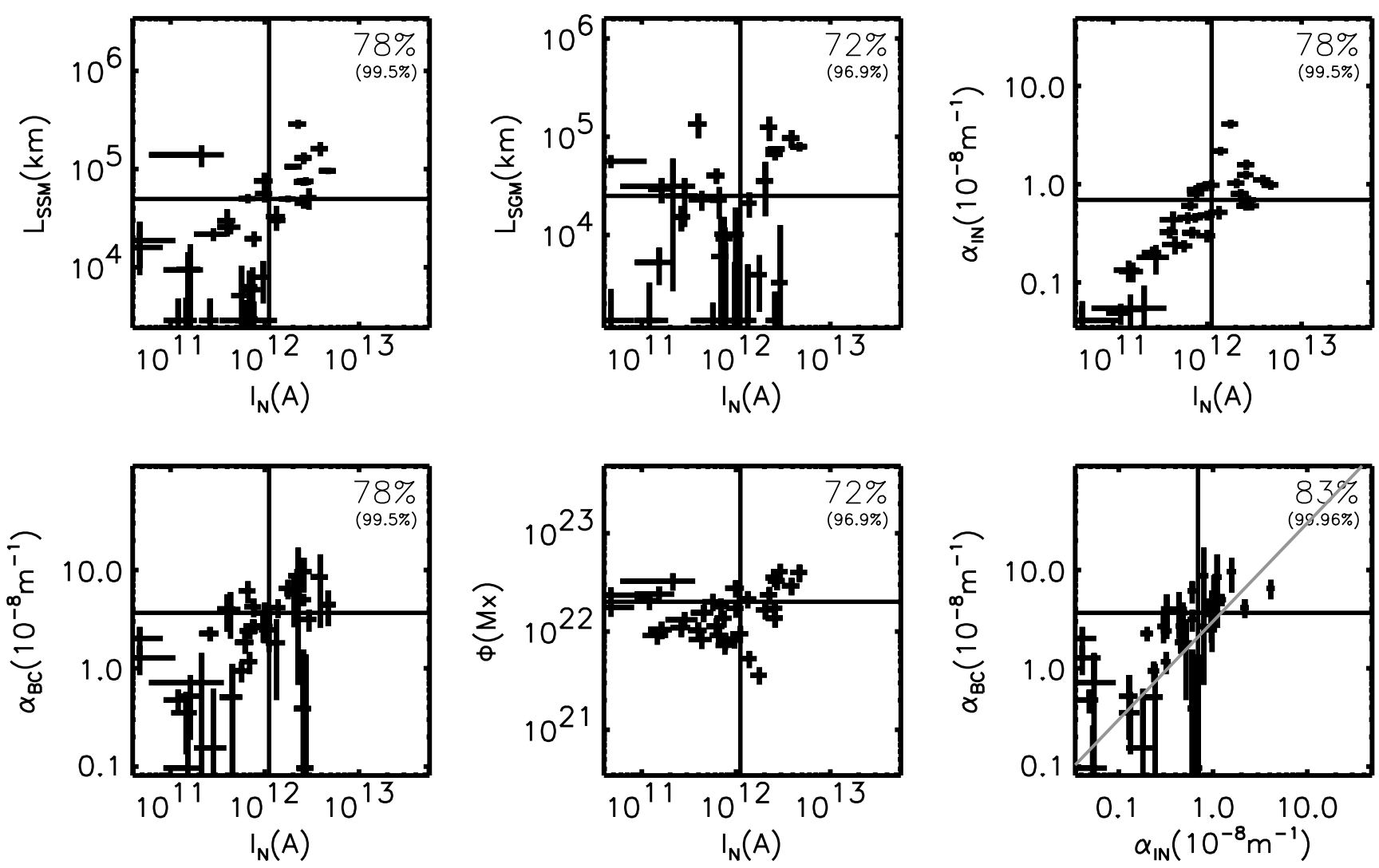

FIG. 2.-Correlation plots of six pairs of magnetic measures from the deprojected magnetograms. In each panel, a pair of measures is plotted on a log-log plot. The pair of measured values form each magnetogram is plotted as a cross with the $1 \sigma$ uncertainty in each value given by the corresponding span of the cross. The adopted thresholds ( Table 1) are plotted as vertical or horizontal lines. The first five panels have the other measures plotted vs. the net current. In the last panel, the two measures of magnetic twist are plotted against each other. In the top right corner of each panel the percentage in larger font is the success rate of the correlation and the percentage in parentheses is the confidence level of the correlation. The diagonal line in the last plot is $\alpha_{\mathrm{BC}}=3 \alpha_{\mathrm{IN}}$, which fits the observations (see text).

There is no a priori way to set the threshold. In Falconer et al. (2002) the adopted threshold was the median value of each measure. This was appropriate because nearly half of the active regions were CME productive. The 19 new active regions added to the sample are on average less nonpotential and less CME productive than the original 12 active regions. So we now allow the data to set the threshold, as we did in Falconer et al. (2003). In the 36 magnetogram sample, only 12 of the magnetograms (33\%) are from active regions that produced a CME during the \pm 2 day window. Thus, our threshold for each measure is set so that $33 \%$ (12 of 36) of our magnetograms have that measure equal to or greater than the threshold. That is, for each measure the threshold value is the 12th largest value in the set of 36 values of that measure obtained from the 36 magnetograms. The \pm 2 day window was chosen for setting thresholds because Falconer et al. (2002a) found that the correlations were strongest for each measure for this window. Setting the threshold this way permits the correlation of a measure with CME productivity in the \pm 2 day window in our sample to have a CME-prediction success rate as high as $100 \%$. The adopted thresholds for each measure is given in Table 1, and each measure equal to or greater than the adopted threshold is marked by an asterisk.

\section{ANALYSIS}

\subsection{Correlations of Measures from Deprojected Magnetogram}

We have three categories of active-region magnetogram measures: measures of total nonpotentiality $\left(I_{N}, L_{\mathrm{SSM}}\right.$, and $\left.L_{\mathrm{SGM}}\right)$, measures of overall magnetic twist $\left(\alpha_{\mathrm{IN}}\right.$ and $\left.\alpha_{\mathrm{BC}}\right)$, and a mea- sure of the size of an active region $(\Phi)$. Measures of the same category are expected to show a strong correlation with each other. Measures of different categories could in principle be uncorrelated, correlated, or anticorrelated. It is reasonable to expect a positive correlation of each total-nonpotentiality measure with the twist measures and with the size measure. Consider two active regions of different size that have the same magnetic twist (same size-normalized nonpotentiality); the larger active region will have a larger $I_{N}$ than the smaller active region. The magnetic shear along the main neutral line is an aspect of the overall magnetic twist in the active region's magnetic field. Because the larger active region has longer main neutral line and the two active regions both have the same fraction of the main neutral line that is strongly sheared (or that has strong gradient), the larger active region will have larger $L_{\mathrm{SSM}}$ (and larger $L_{\mathrm{SGM}}$ ). There is no a priori reason to expect that the magnetic twist measures will be correlated with active-region size. If a positive (negative) correlation were found, this would indicate that the Sun has a tendency to produce more twist in large active regions than in small active regions (or the reverse) by some mechanism.

To examine the correlations between measures, we plot selected pairs of measures as shown in Figure 2. Table 2 lists the success rates and confidence levels of the correlations. The confidence level or statistical significance of a correlation is $(1-P) \times 100 \%$, where $P$ is the probability that a correlation as strong or stronger than the observed correlation could have occurred by random chance. A correlation having a confidence level $\geq 95 \%$ is conventionally considered to be statistically significant (Everitt 1977). The success rate is the percent of the sample 
TABLE 2

Correlation Confidence Levels and Success Rates of the Pairs of our Magnetic Measures from Deprojected Magnetograms

\begin{tabular}{|c|c|c|c|c|c|}
\hline Quantity & $L_{\mathrm{SSM}}$ & $L_{\mathrm{SGM}}$ & $\alpha_{\mathrm{IN}}$ & $\alpha_{\mathrm{BC}}$ & $\Phi$ \\
\hline$I_{N}$ & $77 \pm 6(99.5)$ & $72 \pm 6(96.9)$ & $77 \pm 6(99.5)$ & $77 \pm 6(99.5)$ & $72 \pm 6(96.9)$ \\
\hline$L_{\mathrm{SSM}}$ & $\ldots$ & $77 \pm 6(99.5)$ & $61 \pm 6(65)$ & $66 \pm 6(86)$ & $77 \pm 6(99.5)$ \\
\hline$L_{\mathrm{SGM}}$ & $\ldots$ & $\ldots$ & $61 \pm 6(65$ & $66 \pm 6(86)$ & $72 \pm 6(96.9)$ \\
\hline 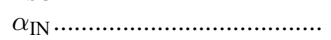 & $\ldots$ & $\ldots$ & $\ldots$ & $83 \pm 6(99.96)$ & $50 \pm 6(16)$ \\
\hline$\alpha_{\mathrm{BC}} \ldots \ldots$ & $\ldots$ & $\ldots$ & $\ldots$ & $\ldots$ & $50 \pm 6(16)$ \\
\hline
\end{tabular}

Noтеs.-The first number is the success rate in percent, the rate at which the two measures agree in classification relative to the adopted thresholds. The number in parentheses is the correlation confidence level, also in percent.

for which the two measures are both equal to or above threshold or both below threshold. For our sample size of 36 magnetograms, the minimum step size of success rate is $6 \%$. Differences of this size among different pairs of measures we consider to be insignificant. Details about confidence levels, success rate, and step size are presented in the Appendix. Our 36 magnetogram sample is only large enough to show a statistically significant correlation between a pair of measures that has a correlation success rate greater than about $70 \%$ (see the Appendix). For our sample size, weaker correlations (having success rates less than about $70 \%$ ) cannot be shown to be statistically significant.

As expected, the three total-nonpotentiality measures $\left(I_{N}, L_{\mathrm{SSM}}\right.$, and $\left.L_{\mathrm{SGM}}\right)$ are correlated at statistically significant levels, and these correlations have good success rates $(\sim 75 \%)$. The two magnetic-twist measures $\left(\alpha_{\mathrm{IN}}, \alpha_{\mathrm{BC}}\right)$ have a statistically significant correlation with a comparably high success rate $(83 \%)$. For most active regions the value of $\alpha_{\mathrm{IN}}$ is lower than that for $\alpha_{\mathrm{BC}}$. The adopted threshold of $\alpha_{\mathrm{IN}}$ is a factor of 3 smaller than for $\alpha_{\mathrm{BC}}$. In the plot of $\alpha_{\mathrm{BC}}$ versus $\alpha_{\mathrm{IN}}$ in Figure 2, the line $\alpha_{\mathrm{BC}}=3 \alpha_{\mathrm{IN}}$ roughly fits the distribution. The factor of 3 mostly arises from the net current being only the current in either the leading or trailing polarity, while the total magnetic flux is for the entire active region, both positive and negative flux.

The correlations between different categories of measures (Table 2) are consistent with the total (non-size-normalized) nonpotentiality of an active region being the product of two uncorrelated or nearly uncorrelated quantities: the degree of overall magnetic twist (size-normalized nonpotentiality) of the active region and the size of the active region. That the success rates of the correlations of the twist measures with the size measure are only $\sim 50 \%$ means that the degree of twist and the size of active regions are nearly if not completely uncorrelated. That size and twist are uncorrelated is consistent with the total nonpotentiality, the product of size and twist, being nearly equally correlated with both the size measure and the twist measures (success rates $\sim 75 \%$ for size and $\sim 70 \%$ for twist). The nature of the correlation between total nonpotentiality and size can be seen best in the plot of $\Phi$ versus $I_{N}$ in Figure 2, where for active regions with small net current there appears to be minimal correlation with size, while for active regions with large net currents there is an absence of small flux active regions. In other words, to have an active region with large net current two conditions are needed: large current density (large magnetic twist) and large area. A small active region for a current density in the range observed in our sample cannot have a large net current.

\subsection{Correlation of Measures from Deprojected Magnetograms with Active-Region CME Productivity}

For each of the six active-region magnetic measures from deprojected magnetograms, Figure 3 shows the correlation with the CME productivity of the active regions in the time window spanning \pm 2 days from the day of the magnetogram. The success rates and confidence levels of these correlations and of the correlations for the \pm 1 day and \pm 4 day windows are listed in Table 3 .

Compatible with the correlations found for the 17 magnetogram sample of Falconer et al. (2002a), Table 3 shows a slight tendency for the correlations of our measures with CME productivity to be stronger for the \pm 2 day window than for either the \pm 1 day window or the \pm 4 day widow. This tendency is only marginally discernible in Table 3 : for any one measure, the
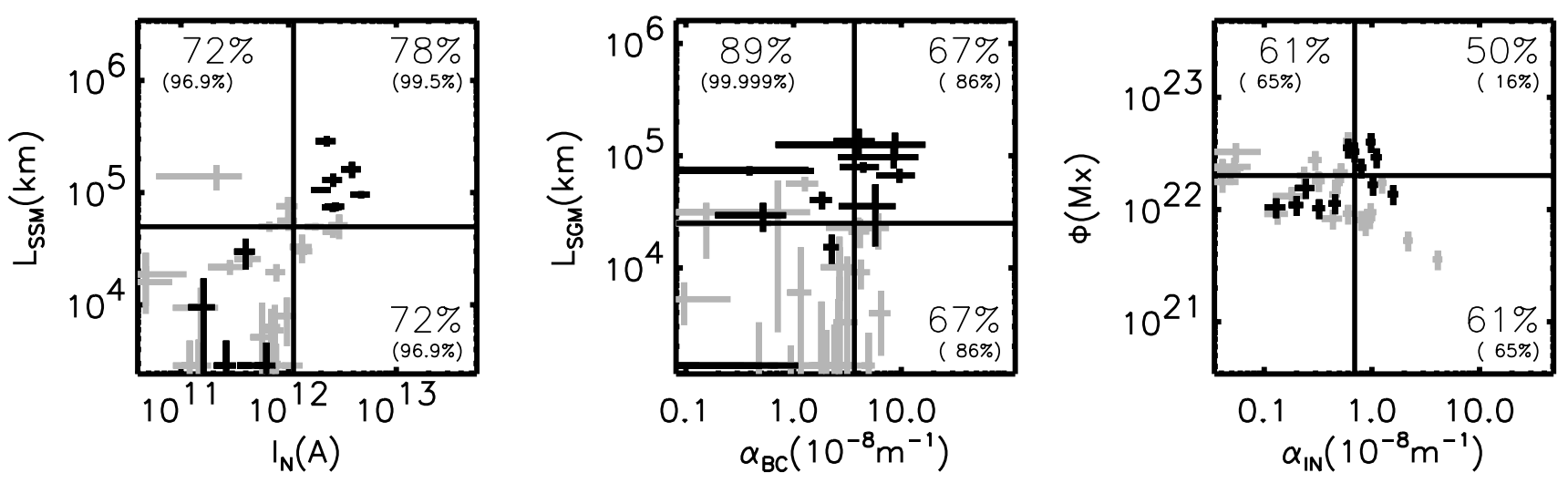

FIG. 3.-Correlation of magnetic measures from the deprojected magnetograms with active-region CME productivity in the \pm 2 day window. Each panel shows one

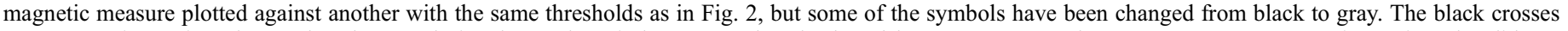

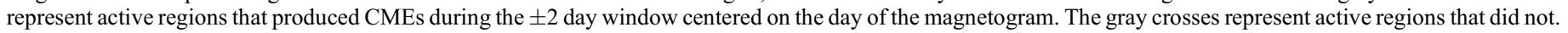

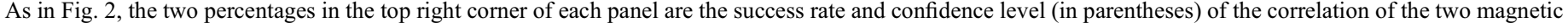

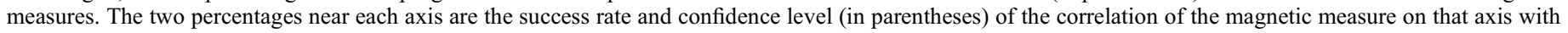
active-region CME productive in the \pm 2 day window. 
TABLE 3

Correlation of CME Productivity with Magnetic Measures From Deprojected Magnetograms

\begin{tabular}{|c|c|c|c|}
\hline Quantity & \pm 1 Days & \pm 2 Days & \pm 4 Days \\
\hline$L_{\mathrm{SSM}} \cdot \cdot$ & $69 \pm 6(88)$ & $72 \pm 6(96.9)$ & $66 \pm 6(93.8)$ \\
\hline$L_{\mathrm{SGM}} \ldots \ldots \ldots \ldots \ldots \ldots \ldots \ldots \ldots \ldots \ldots \ldots \ldots$ & $80 \pm 6(99.8)$ & $88 \pm 6(99.999)$ & $77 \pm 6(99.9)$ \\
\hline$I_{N} \ldots \ldots \ldots \ldots \ldots \ldots \ldots \ldots \ldots$ & $69 \pm 6(88)$ & $72 \pm 6(96.9)$ & $77 \pm 6(99.9)$ \\
\hline$\alpha_{\mathrm{IN}} \cdots$ & $58 \pm 6(35)$ & $61 \pm 6(65)$ & $55 \pm 6(54)$ \\
\hline$\alpha_{\mathrm{BC}} \cdots$ & $63 \pm 6(66)$ & $66 \pm 6(86)$ & $66 \pm 6(93.8)$ \\
\hline$\Phi$ & $58 \pm 6(35)$ & $61 \pm 6(65)$ & $61 \pm 6(79)$ \\
\hline
\end{tabular}

Note.-The first number is the correlation's success rate in percent; the number in parentheses is the correlation's confidence level in percent.

differences in success rates among the three windows are most often less than 1 step (6\%) and always less than 2 steps (12\%). That is, over this range of centered time windows, the strength of the correlation of each of the six measures with CME productivity depends at most only weakly on the span of the window.

In contrast to the slight changes in the correlation strength between windows for any one measure in Table 3 , there is a more significant difference between the total-nonpotentiality measures and the other two categories of measures. For the total nonpotentiality measures $\left(L_{\mathrm{SSM}}, L_{\mathrm{SGM}}\right.$, and $\left.I_{N}\right)$ success rates of the correlation with CME productivity are all $\sim 75 \%$ (all are within about 2 steps of this value), whereas the success rates of the correlations for the magnetic-twist measures $\left(\alpha_{\mathrm{IN}}, \alpha_{\mathrm{BC}}\right)$ and the active-region size measure $(\Phi)$ are all $\sim 65 \%$ (all are within 2 steps of this value). The success rates of $\sim 65 \%$ for the twist and size measures agree with the $\sim 65 \%$ success rates of the correlations found by Canfield et al. (1999): the correlation of active-region sigmoidality (a signature of the overall magnetic twist) with CME productivity and the correlation of active-region sunspot area (a measure of active-region size) with CME productivity. [These two correlations with $\sim 65 \%$ success rates are statistically significant (correlation confidence level $>95 \%$ ) in the Canfield et al. (1999) set of 107 active regions, but a correlation with $65 \%$ success rate cannot be shown to be statistically significant with our sample size of 36 (Fig. 7 in the Appendix below). Our sample size is the reason that these correlations have confidence levels less than 95\% in Tables 3, 4, and 5. However, we still trust the $\sim 65 \%$ success rates of these correlation of our twist and size measures with CME productivity because they agree with the corresponding statistically significant results of Canfield et al. (1999).] In addition, the $\sim 75 \%$ success rate for the total-nonpotentiality measures is consistent with the total nonpotentiality of an active region being proportional to the product of the degree of overall twist in the magnetic field of the active region and the size of the active region: the success rate

TABLE 4

Strength of Magnetic Measures from Deprojected Magnetograms As Predictors of CMEs

\begin{tabular}{|c|c|c|c|}
\hline Quantity & 0-1 Days & 0-2 Days & 0-4 Days \\
\hline$L_{\mathrm{SSM}} \cdots$ & $72 \pm 6(92.0)$ & $75 \pm 6(97.1)$ & $72 \pm 6(95.5)$ \\
\hline$L_{\mathrm{SGM}} \ldots$ & $72 \pm 6(92.0)$ & $75 \pm 6(97.1)$ & $66 \pm 6(82)$ \\
\hline 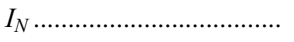 & $72 \pm 6(92.0)$ & $75 \pm 6(97.1)$ & $77 \pm 6(99.3)$ \\
\hline (......................... & $66 \pm 6(69)$ & $69 \pm 6(85)$ & $61 \pm 6(55)$ \\
\hline$\alpha_{\mathrm{BC}} \cdot \cdot$ & $66 \pm 6(69)$ & $69 \pm 6(85)$ & $66 \pm 6(82)$ \\
\hline$\Phi \ldots \ldots \ldots$ & $61 \pm 6(34)$ & $63 \pm 6(57)$ & $66 \pm 6(82)$ \\
\hline
\end{tabular}

NotE.-The first number is the correlation's success rate; the number in parentheses is the correlation's confidence level.
TABLE 5

Strength of Magnetic Measures from Non-deprojected Magnetograms as Predictors of CMEs

\begin{tabular}{|c|c|c|c|}
\hline Quantity & 0-1 Days & 0-2 Days & 0-4 Days \\
\hline${ }^{n} L_{\mathrm{SSM}} \cdots \cdots$ & $72 \pm 6(92)$ & $75 \pm 6(97)$ & $72 \pm 6(95)$ \\
\hline${ }^{n} L_{\mathrm{SGM}} \ldots \ldots \ldots \ldots \ldots \ldots \ldots \ldots \ldots \ldots \ldots \ldots \ldots \ldots \ldots$ & $78 \pm 6(99)$ & $81 \pm 6(99.7)$ & $78 \pm 6(99.3)$ \\
\hline 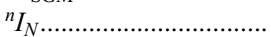 & $72 \pm 6(92)$ & $75 \pm 6(97)$ & $72 \pm 6(95)$ \\
\hline 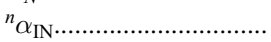 & $78 \pm 6(99)$ & $81 \pm 6(99.7)$ & $72 \pm 6(95)$ \\
\hline${ }^{n} \alpha_{\mathrm{BC}} \ldots .$. & $72 \pm 6(92)$ & $81 \pm 6(99.7)$ & $72 \pm 6(95.5)$ \\
\hline${ }^{n} \Phi$ & $61 \pm 6(34)$ & $64 \pm 6(57)$ & $67 \pm 6(82)$ \\
\hline
\end{tabular}

Note.-The first number is the correlation's success rate; the number in parentheses is the correlation's confidence level.

for the product should be greater than the success rate for either of the two independent factors. Thus, the correlations of our active-region magnetic measures with CME productivity indicate that the total nonpotentiality (total free magnetic energy) of an active region is a stronger determinant of the active region's CME productivity than is the active region's degree of overall magnetic twist (helicity) alone.

\subsection{Evaluation of Measures from Deprojected Magnetograms as CME Predictors}

As was shown in the preceding section, each of our six activeregion magnetic measures has a positive correlation with activeregion CME productivity in time windows of 3-9 days centered on the day of the magnetogram. For the total-nonpotentiality measures, these correlations are strong enough (success rate $\sim 75 \%$ ) to be shown at a statistically significant level (correlation confidence level $>95 \%$ ) by a sample the size of ours (36 activeregion magnetograms), but, for the twist and size measures, the correlations are not strong enough (success rate $\sim 65 \%$ ) to be shown at a statistically significant level by a sample of this size (see the Appendix). For forward time windows corresponding to our three centered time windows, namely $0-1,0-2$, and 0-4 days, it is reasonable to expect that for each measure the correlation with active-region CME productivity should be about as strong as it is for the centered windows. We have examined these forward-time-window correlations to check the veracity of this expectation. These correlations for the $0-2$ day window are shown in Figure 4. For each of the three forward time windows, the success rates and confidence levels of the correlations are listed in Table 4. The success rate of the correlation of a measure with active-region CME productivity in a forward window is the success rate of that measure as a predictor of CME production by an active region during that span of the future.

As expected, the correlation success rate and confidence levels for the forward time widows ( Table 4) are about the same as those for the centered windows (Table 3). As for the centered windows, for the forward windows the success rates of the correlations of the total nonpotentiality measures with CME productivity are $\sim 75 \%$ and the success rates of the correlations of the magnetic-twist and size measures with CME productivity are $\sim 65 \%$. This result again agrees with the total nonpotentiality of an active region being proportional to the product of the active region's overall magnetic twist and size, and it again indicates that the total free magnetic energy in an active region's magnetic field is a stronger predictor of the active region's CME productivity than is the overall magnetic twist alone.

The results in Table 4 are also similar to those in Table 3 in that the correlations of the measures with CME productivity tend to be slightly stronger for the 0-2 day window than for 

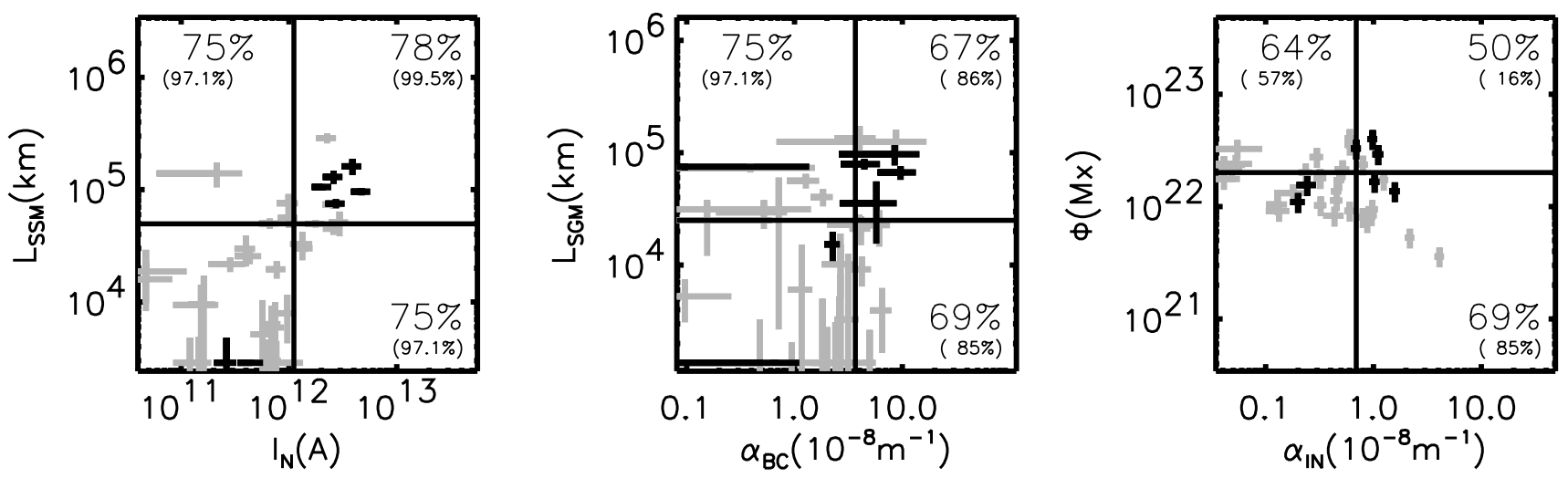

FIG. 4.-Performance of magnetic measures from deprojected magnetograms as predictors of active-region CME productivity. This figure is of the same format as Fig. 3, with black crosses indicating active regions that produced at least one CME during the $0-2$ day window. The gray crosses are for active regions that did not produce a CME in this window. Compared to Fig. 3, there are more gray crosses beyond the threshold; the additional gray crosses are active regions that produced CMEs only in the first two days of the \pm 2 day window.

either the $0-1$ day window or the $0-4$ day window. As noted in $\S 2.4$ (after Falconer et al. 2002a), this tendency is plausibly explained as resulting from the combination of two characteristics of active regions: (1) the rate of CME production by the most strongly nonpotential active regions is usually no more than about one per day, and (2) the timescale for the buildup or decay of high levels of nonpotentiality in an active region is typically several days.

The results in Table 4 also show that, for active regions at disk center, the measure of total nonpotentiality, $L_{\mathrm{SGM}}$, which can be measured from a line-of-sight magnetogram, is as strong a predictor of CME production as the other two measures of total nonpotentiality, $L_{\mathrm{SSM}}$ and $I_{N}$, which can only be measured from a vector magnetogram.

\subsection{Evaluation of Measures from Non-deprojected Magnetograms as Approximations of Measures from Deprojected Magnetograms and as CME Predictors}

For each of our six active-region magnetic measures, the correlation of the values measured from the non-deprojected
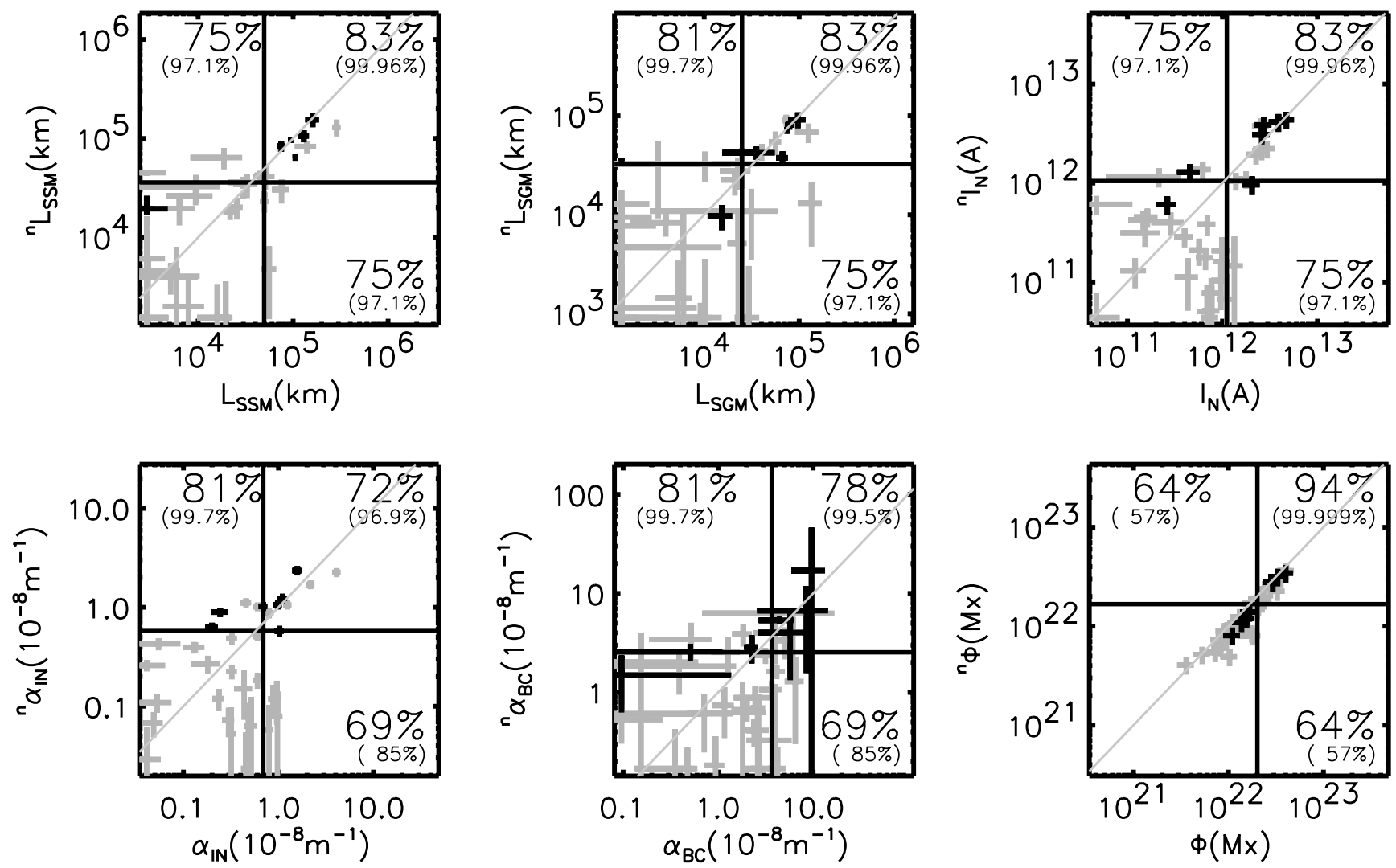

FIG. 5.-Performance of measures from non-deprojected magnetograms as approximations of corresponding measures from deprojected magnetograms and as

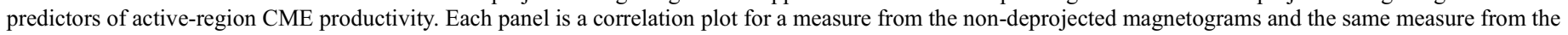

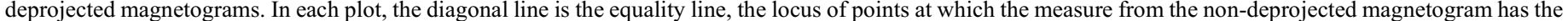

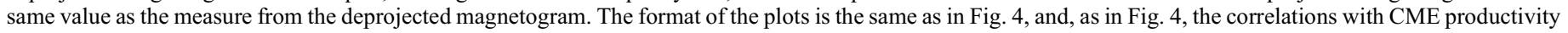
are for the 0-2 day window. 
magnetograms with the values measured from the deprojected magnetograms is shown in Figure 5. For each measure, the plot in Figure 5 also gives for the measure from the non-deprojected magnetograms and for the measure from the deprojected magnetograms the success rate and confidence level of the correlation with active-region CME productivity in the $0-2$ day window. For the measures from the non-deprojected magnetograms, the success rates and confidence levels of the correlations with $\mathrm{CME}$ productivity in the $0-1,0-2$, and $0-4$ day windows are listed in Table 5.

First, consider the correlations of the measures from the nondeprojected magnetograms with the corresponding measures from the deprojected magnetograms. The measure having the strongest correlation is the total magnetic flux ${ }^{n} \Phi(94 \%$ success rate; Fig. 5). The measures of total nonpotentiality $\left({ }^{n} L_{\mathrm{SSM}},{ }^{n} L_{\mathrm{SGM}}\right.$, ${ }^{n} I_{N}$ ) have the next strongest correlation ( $83 \%$ success rate), while the measures of overall magnetic twist $\left({ }^{n} \alpha_{\mathrm{IN}},{ }^{n} \alpha_{\mathrm{BC}}\right)$ have the weakest correlation ( $\sim 75 \%$ success rate). For each of the measures of total nonpotentiality and for each of the measures of magnetic twist, the scatter in the correlation plot is much greater for the weakly nonpotential active regions than for the strongly nonpotential active regions (Fig. 5). In contrast, the scatter in the correlation plot for the magnetic flux content is strikingly less than for the other five measures, and the scatter for smaller active regions is about the same as that for larger active regions. In addition, whereas for the other five measures the scatter is roughly symmetric about the diagonal line of equality, for the flux content, while the points hug the equality line more closely, they noticeably tend to fall below that line (Fig. 5).

The above contrasting trends can be understood as follows. First, the scatter in the correlation plot for the flux content $(\Phi$, $\left.{ }^{n} \Phi\right)$ is mostly from a simple projection effect. If the magnetic field in active regions is predominately vertical on average over the active region, then the strength of the line-of-sight component of the magnetic field and the apparent area of the active region both decrease as $1 / \cos \theta$, where $\theta$ is the average angle between the line-of-sight and the vertical at the location of the active region. Upon multiplying the values of ${ }^{n} \Phi$ measured from the non-deprojected magnetograms by the $\left(1 / \cos \theta^{2}\right)$ correction factor for each active region, we obtain the correlation plot in Figure 6. This plot has less scatter than the corresponding plot in Figure 5, and the scatter is much more nearly symmetric about the equality line. Thus, the scatter in the correlation plot for flux content in Figure 5 is well understood.

Now, in Figure 5, why do the correlation plots for the five measures of active-region nonpotentiality $\left({ }^{n} L_{\mathrm{SSM}},{ }^{n} L_{\mathrm{SGM}},{ }^{n} I_{N}\right.$, ${ }^{n} \alpha_{\mathrm{IN}},{ }^{n} \alpha_{\mathrm{BC}}$ ) all have scatter that differs from that of the correlation plot for active-region flux content $\left({ }^{n} \Phi\right)$ in being (1) markedly greater, (2) more symmetric about the equality line, and (3) much greater for active regions below both CME-expectation thresholds than for active regions above both thresholds? We surmise that, even for active regions within $\sim 30^{\circ}$ of disk center, projection effects produce much larger artifacts in magnetic twist and shear than in the magnetic flux. The symmetry of the scatter about the equality line in the plots for the nonpotentiality measures indicates that the artifacts in the magnetic shear and twist can produce either positive or negative errors in the nonpotentiality measures, depending on the orientation and magnetic configuration of the active region. For active regions within $\sim 30^{\circ}$ of disk center, the magnitudes of the projection errors in the nonpotentiality measures apparently are comparable to those of our CME expectation thresholds. As a result, in the log-log plots, there is larger scatter in the bottom left quadrant than in the top right quadrant. Finally, because (1) the total nonpotentiality of

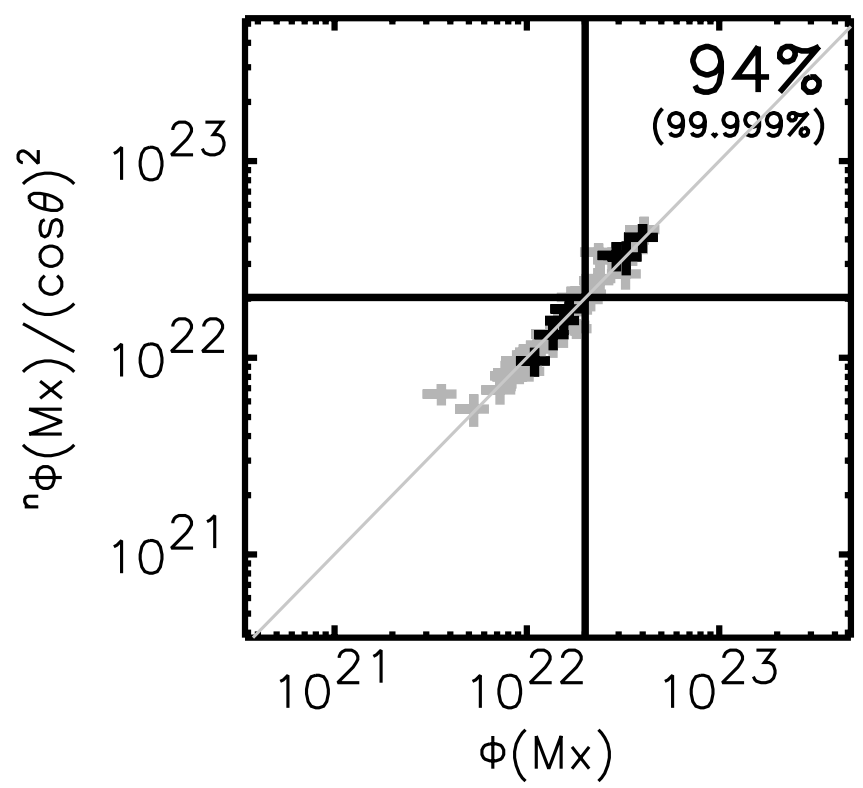

FIG. 6.-Correlation of active-region flux content ${ }^{n} \Phi /(\cos \theta)^{2}$ measured and corrected from our 36 non-deprojected magnetograms with active-region flux content $\Phi$ measured from the deprojected magnetograms. Compared to the correlation plot of ${ }^{n} \Phi$ and $\Phi$ in Fig. 5, the scatter is reduced and shifted up to be more nearly symmetric about the diagonal line of equality.

an active region is the product of its size and its overall magnetic twist and/or shear and (2) the projection errors in ${ }^{n} \Phi$ are much smaller than the projection errors in the twist measures ${ }^{n} \alpha_{\mathrm{IN}}$ and ${ }^{n} \alpha_{\mathrm{BC}}$, it is reasonable that the strengths of the correlations for the total-nonpotentiality measures $(83 \%$ success rate) are intermediate between the strengths of the correlations for the magnetic-twist measures ( $\sim 75 \%$ success rate) and the strength of the correlation for the flux content ( $94 \%$ success rate).

We now turn to the correlation of the measures from the nondeprojected magnetograms with active-region CME productivity (Fig. 5, Table 5). For the measures of total nonpotentiality, as predictors of active-region CME productivity, the values from the non-deprojected magnetograms have the same success rate as the values from the deprojected magnetograms $(\sim 75 \%)$. Likewise, for the magnetic flux content, as a predictor of CMEs, the values of ${ }^{n} \Phi$ have the same success rate as the values of $\Phi(\sim 65 \%)$. This means that for active-regions within $\sim 30^{\circ}$ of disk center, for CME forecasting, adequate measures of active-region total nonpotentiality and total magnetic flux can be obtained from non-deprojected magnetograms (even though sizeable errors are produced in the measures of total nonpotentiality by projection effects). Of particular importance, adequate measures of $L_{\mathrm{SGM}}$ and $\Phi$ can be obtained from line-of-sight magnetograms of active regions within $\sim 30^{\circ}$ of disk center, such as from the full-disk magnetograms from SOHO MDI (whose active-region magnetograms cannot be deprojected to disk center).

In contrast to the flux content and the measures of total nonpotentiality, for our sample of 36 active-region magnetograms, the magnetic-twist measures from the non-deprojected magnetograms do not have the same CME-prediction success rate as the corresponding measures from the deprojected magnetograms (Fig. 5, Table 5). Instead, the values measured from the nondeprojected magnetograms have a CME-prediction success rate ( $\sim 75 \%)$ that is about 2 steps higher than from the values measured from the deprojected magnetograms $(\sim 65 \%)$. In view of the large errors produced in ${ }^{n} \alpha_{\mathrm{IN}}$ and ${ }^{n} \alpha_{\mathrm{BC}}$ by projection effects, we consider the higher success rate for the values from 
the non-deprojected magnetograms to be spurious result. A similar result (which in hindsight we now consider to be spurious) was found by Falconer et al. (2002a) from 17 non-deprojected magnetograms: ${ }^{n} \alpha_{\mathrm{IN}}$ was found to be as strongly correlated as ${ }^{n} L_{\mathrm{SSM}}$ and ${ }^{n} I_{N}$ with active-region CME productivity. The reason that the CME-prediction success rate of the total-nonpotentiality measures from non-deprojected magnetograms remains unchanged from that from deprojected magnetograms while the success rate of the twist measures from the non-deprojected magnetograms deviates from that from the deprojected magnetograms is plausibly that, because the total nonpotentiality is proportional to the flux content whereas the twist measures are independent of the flux content, ${ }^{n} \Phi$ and its small projection errors give the total-nonpotentiality measures from the non-deprojected magnetograms a correlation with the CME productivity that is more accurate than that for the twist measures from the non-deprojected magnetograms. From the above results and considerations, we conclude that for the purpose of examining the importance of magnetic twist relative to the importance of active-region total nonpotentiality and size in causing CMEs, adequate values of our measures of overall magnetic twist can only be obtained from deprojected magnetograms, even for active regions within $\sim 30^{\circ}$ of disk center.

\section{SUMMARY AND DISCUSSION}

For a sample of $36 \mathrm{MSFC}$ vector magnetograms of basically bipolar active regions, we measured six whole-active-region magnetic quantities from each magnetogram, once from the original (non-deprojected) plane-of-the-sky magnetogram, and again after deprojection of the magnetogram to disk center. Each of the quantities measured from a deprojected magnetogram is a definite physical quantity; each corresponding quantity measured from the non-deprojected magnetogram is an approximation of that physical quantity. Three of the six active-region measures are measures of the total nonpotentiality of the active region; these are the length of strong-shear main neutral line $\left(L_{\mathrm{SSM}}\right)$, the length of stronggradient main neutral line $\left(L_{\mathrm{SGM}}\right)$, and the net vertical electric current $\left(I_{N}\right)$. Two others are measures of the degree of overall twist in the active region's magnetic field (each reflects the twist in the magnetic field in the manner of the $\alpha$ parameter in a forcefree magnetic field); these are the net-current alpha $\left(\alpha_{\mathrm{IN}}\right)$ and the best constant alpha $\left(\alpha_{\mathrm{BC}}\right)$. The sixth magnetic measure is a measure of the size of the active region; it is the flux content, the total magnetic flux $(\Phi)$ in the active region. The measures of magnetic twist are intrinsically independent of the size of the active region; they are measures of the active region's nonpotentiality normalized by the flux content; that is, they are measures of the nonpotentiality per unit flux. The total nonpotentiality of an active region is the product of the flux content and the nonpotentiality per unit flux. Hence, we expect our measures of total nonpotentiality to be roughly proportional to the product of the flux content and either of our magnetic-twist measures.

With our six magnetogram measures, we investigated the magnetic causes of active-region CMEs by examining three sets of correlations for the measures from the deprojected magnetograms: (1) the correlations of the measures with each other, (2) the correlations of the measures with the CME productivity of the active regions in windows of a few days centered on the day of the magnetogram, and (3) the correlations of the measures with CME productivity in windows of a few days extending forward from the day of the magnetogram. We assessed the six measures from the non-deprojected magnetograms as proxies for the measures from the deprojected magnetograms by examining two sets of correlations: (1) the correlation of each of the measures from the non-deprojected magnetograms with the corresponding measure from the deprojected magnetograms, and (2) the correlations of the measures from the non-deprojected magnetograms with $\mathrm{CME}$ productivity in the forward-looking windows.

All of the above correlations were found to be consistent with each of our total-nonpotentiality measures being a measure of a quantity that increases monotonically with the product of the overall magnetic twist and the magnetic flux content of the active region. In particular, from the correlations among the measures, we found that (1) all of the five measures of nonpotentiality were correlated with each other, and (2) the correlations of the totalnonpotentiality measures with flux content were comparably strong, but (3) in contrast, there was no discernible correlation of the magnetic-twist measures with flux content. These results agree with the total nonpotentiality (total free magnetic energy) of an active region being roughly the product of the overall twist and the flux content of its magnetic field.

Each of the six active-region magnetic measures from the deprojected magnetograms had a positive correlation with activeregion CME productivity in each of the centered windows $( \pm 1$, $\pm 2, \pm 4$ days) and in each of the forward windows (0-1, $0-2$, 0-4 days). The total-nonpotentiality measures were more strongly correlated with CME productivity than were either the magnetictwist measures or the flux content: the success rates were $\sim 75 \%$ for the total-nonpotentiality measures and $\sim 65 \%$ for the other measures. The $\sim 65 \%$ success rates for the magnetic-twist measures and for the active-region size measure, total magnetic flux, match the $\sim 65 \%$ success rates for the correlations (found by Canfield et al. 1999) of active-region eruptive-flare productivity with the overall magnetic twist (sigmoidality) and magnetic size (sunspot area) of active regions. Together with the absence of correlation between the magnetic-twist measures and the flux content, these results indicate that the magnetic twist and flux content each are separate but comparably strong causes of CME production. That the total-nonpotentiality measures are stronger predictors of CME productivity than are either the magnetic-twist measures or the flux content is compatible with the total nonpotentiality of an active region being proportional to the product of the overall magnetic twist and the flux content of the magnetic field. Moreover, that the total-nonpotentiality measures are stronger predictors of CME productivity than are the magnetic-twist measures is evidence that the total free magnetic energy in an active region's magnetic field is a stronger determinant of the CME productivity of the active region than is the magnetic field's overall twist (or large-scale helicity) alone. This observational result agrees with recent modeling results of Phillips et al. (2005) that also favor the magnetic field's free energy over its helicity as the main determinant for CME production.

It is obvious that the magnetic twist should be positively correlated with active-region CME productivity: if there were no twist, the field would be in its minimum-energy potential configuration and could not explode to produce a CME. It is not so obvious why the magnetic size of an active region should have a positive effect on CME productivity, or why magnetic size should be about as strong as magnetic twist as a cause of CMEs. One possibility is that most active regions are significantly hemmed in by surrounding magnetic fields, so that larger and stronger active regions are more able to overcome this restraint to the production of the ejective explosions that become CMEs. Another possibility is that smaller scale aspects of an active region's magnetic field are important for the production of CMEs, that these aspects are not reflected in our measures of overall magnetic twist, and that these aspects are more prevalent in larger active regions. 
We found that the measures of active-region total nonpotentiality and flux content from the non-deprojected magnetograms were as strongly correlated with CME productivity as were the corresponding measures from the deprojected magnetograms. This indicates that, for active regions within $30^{\circ}$ of disk center, adequate measures of total nonpotentiality and flux content can be obtained from the $\mathrm{SOHO}$ MDI full-disk line-of-sight magnetograms for further studies of the magnetic causes of CMEs, such as the studies sketched in the following.

We have corroborated Canfield et al. (1999) in finding the overall magnetic twist and the magnetic size of active regions each to be correlated with active-region CME productivity with a success rate of $\sim 65 \%$. The nearly equal strength of the correlations of $\mathrm{CME}$ productivity with magnetic twist and magnetic size, together with the absence of correlation between twist and size, suggests that the product of magnetic twist and magnetic size is close to the combination of twist and size in a single parameter that would have the strongest CME-productivity correlation from both effects. That is, this suggests that our totalnonpotentiality measures might be nearly optimum magnetic measures for forecasting CME productivity to the extent that the CME productivity of active regions is caused by their magnetic twist and magnetic size. However, because of the roughly $\pm 6 \%$ uncertainty in the success rates of correlations for a sample of 36 magnetograms, it remains to be seen from a larger sample whether there is some combination of twist and size (e.g., $\alpha_{\mathrm{IN}}$ or $\alpha_{\mathrm{BC}}$ times $\Phi^{\gamma}$, where $\gamma$ is appreciably larger or smaller than unity) that gives a magnetic measure that is a significantly better CME predictor than our measures of total nonpotentiality. That is, there is conceivably an appreciable further effect of magnetic size (either positive or negative) on CME productivity, in addition to its effect through the total nonpotentiality. We plan to investigate this question by applying discriminant analysis similar to that of Leka \& Barnes (2003) to the total flux ${ }^{n} \Phi$ and a measure of total nonpotentiality similar to ${ }^{n} L_{\mathrm{SGM}}$ measured from a large sample of $\sim 100$ active-region magnetograms from $\mathrm{SOHO}$ MDI. Effectively, we will examine the correlation of the measure ${ }^{n} L_{\mathrm{SGM}}{ }^{n} \Phi^{\gamma}$ with active-region CME productivity for many values of $\gamma$, covering the range $-1 \leq$ $\gamma \leq 1$. If the correlation success rate is not greatest for $\gamma=0$, but significantly greater for some positive or negative value of $\gamma$, this will mean that the effect of active-region magnetic size on active-region CME productivity is somewhat stronger or weaker than the linear proportionality that would be the case if the correlation of ${ }^{n} L_{\mathrm{SGM}}{ }^{n} \Phi^{\gamma}$ with CME productivity is strongest for $\gamma=0$. If the correlation is strongest for $\gamma=0$, this will indicate that total-nonpotentiality measures such as ours are effectively the optimum combination of the overall magnetic twist and magnetic size for forecasting an active region's CME productivity, and further suggest that the free magnetic energy in an active region is the main determinant of the active region's CME productivity.

The sample of active-region magnetograms measured in the present study is for grossly bipolar active regions. Few nominally bipolar active regions are purely bipolar; instead they have some secondary neutral lines besides the main neutral line. Many active regions are not at all bipolar; instead they are multipolar, having three or more polarity domains of comparable flux content with two or more neutral lines between them. It is therefore of interest to devise a measure of active-region total nonpotentiality that is applicable to multipolar active regions and that includes the secondary neutral lines in nominally bipolar active regions. Our main-neutral line measures, $L_{\mathrm{SSM}}$ and $L_{\mathrm{SGM}}$, are readily generalized to cover all neutral lines in an active region of any magnetic complexity. We plan to extend our study of active-region total nonpotentiality and magnetic flux content as causes of active-region CME productivity by using a generalized version of $L_{\mathrm{SGM}}$. This will allow us to tap the large archive of 16 day $^{-1}$, uniform-quality, full-disk, line-of-sight magnetograms from $\mathrm{SOHO}$ MDI. For a large sample of MDI activeregion magnetograms within $30^{\circ}$ of disk center, along with our generalized measure of total nonpotentiality and the magnetic flux content, we will also measure the rates of change of the total-nonpotentiality measure and flux content. By means of correlation analysis like that in this paper and the bivariate analysis described above, we will investigate (1) whether the generalized measure of total nonpotentiality is as strong a CME predictor for complex active regions as it is for bipolar active regions, and (2) whether any combination of these four measures is more strongly correlated with active-region CME productivity than is the total-nonpotentiality measure alone.

The research reported in this paper was supported by funding from NSF's Division of Atmospheric Sciences through its SHINE and Space Weather programs, and by NASA's Science Mission Directorate through the Living With a Star Targeted Research and Technology program and the Solar and Heliospheric Physics Supporting Research and Technology program of its Heliophysics Division. The paper was improved by several helpful comments from the referee. We acknowledge the use of the CME catalog generated and maintained by the Center for Solar Physics and Space Weather of The Catholic University of America in cooperation with the Naval Research Laboratory and NASA. $S O H O$ is a project of international cooperation between ESA and NASA. Sreeram Balasubramanian, graduate student research assistant, University of Alabama at Huntsville, helped in development and application of computer programs used in calibrating and analyzing the magnetograms.

\section{APPENDIX}

\section{FISHER TEST, SUCCESS RATE, SAMPLE SIZE}

To determine the statistical significance of correlations of a magnetic measure with active-region CME productivity (or of the correlation between two different measures) we apply the following procedure. First, for each magnetogram, we compare each measure to the adopted threshold and determine whether or not the measure is greater than or equal to the adopted threshold (those that are greater than or equal to the threshold are marked by asterisks in Table 1 ). Next, we construct $2 \times 2$ contingency tables as in Table 6 . The probability $P$ that the observed population of the four quadrants or a more unequal distribution would occur by random chance is found by the Fisher Test (Everitt 1977; Falconer et al. 2002a). The confidence level of the correlation is $(1-P) \times 100 \%$. This percentage is listed for each of three example correlations in Table 6 . A correlation having a confidence level greater than $95 \%$ is considered to be statistically significant (Everitt 1977 ).

Besides determining whether a correlation is statistically significant, we determine the success rate of a measure as a predictor of CME productivity (or of whether or not another measure will be greater than or equal to its threshold). This is the percentage of correct 
TABLE 6

EXAMPLES of $2 \times 2$ CONTINGEncy Tables

\begin{tabular}{|c|c|c|c|c|c|c|}
\hline \multirow[b]{2}{*}{ QuANTITY } & \multirow[b]{2}{*}{$I_{N}<$ Threshold } & \multirow[b]{2}{*}{$I_{N} \geq$ ThRESHOLD } & \multicolumn{2}{|c|}{ \pm 2 DAYS } & \multicolumn{2}{|c|}{$0-2$ DAYS } \\
\hline & & & No CMEs & CMEs & No CMEs & CMEs \\
\hline$L_{\mathrm{SSM}} \geq$ Threshold.............. & 4 & 8 & 5 & 7 & 7 & 5 \\
\hline$L_{\mathrm{SSM}}<$ Threshold................ & 20 & 4 & 19 & 5 & 22 & 2 \\
\hline Success Rate $(\%)$ & \multicolumn{2}{|c|}{$77 \pm 6$} & \multicolumn{2}{|c|}{$72 \pm 6$} & \multicolumn{2}{|c|}{$75 \pm 6$} \\
\hline Confidence Level (\%)....... & \multicolumn{2}{|c|}{99.5} & \multicolumn{2}{|c|}{96.9} & \multicolumn{2}{|c|}{97} \\
\hline
\end{tabular}

predictions and is equal to the sum of the populations in the two success quadrants (top right and bottom left quadrants) divided by the sample size times $100 \%$. This success rate is listed in Table 6 for each example correlation. The number after the \pm is the success rate step size. The step size arises from the sums of the rows and columns remaining constant as we compare different measures (i.e., for each measure there are 12 magnetograms that are at or above threshold and 24 that are below threshold in our sample). If one entry in the $2 \times 2$ table changes, the other three also change in ways to keep the sums of the rows and columns constant. This results in the diagonal sums changing in increments of 2 , so that the success rate changes by steps of $(2 / 36) \times 100 \%$ or $\sim 6 \%$.

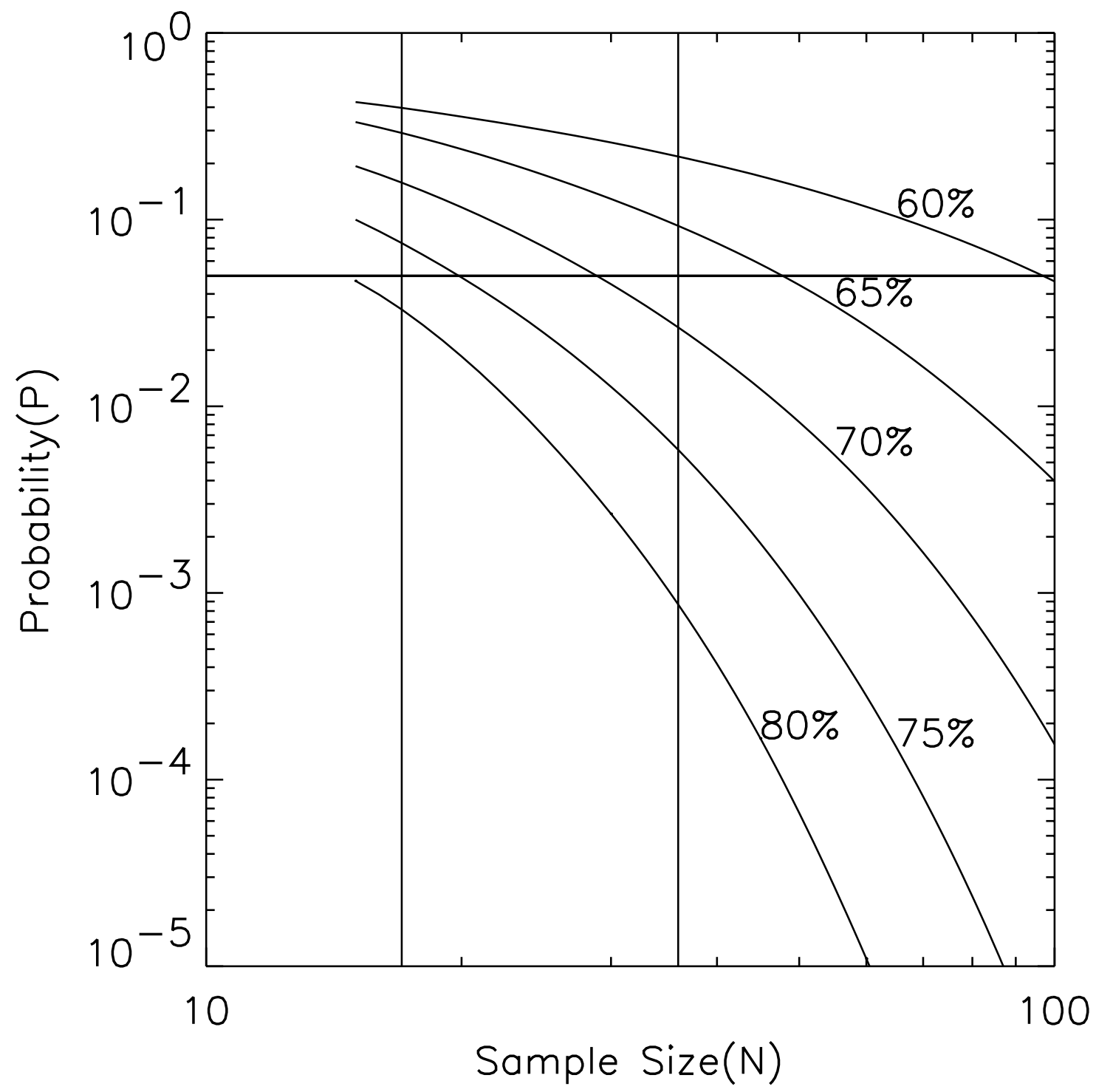

FIG. 7.-Probability of a correlation occurring by random chance, as a function of sample size and success rate. The five curves are for the five labeled success rates. To produce these curves, we evaluated from the Fisher test the probabilities $(P)$ of $2 \times 2$ contingency tables with different sample sizes and success rates. Each table had one-third of the sample greater than or equal to the threshold and two-thirds less than the threshold, and each table had equal populations in its two failure quadrants (top left and bottom right quadrants). The horizontal line is at $P=5 \%$. Below this line the correlation and success rate are considered statistically significant. The vertical lines mark our previous sample size of 17 and our present sample size of 36. A sample of 17 is sufficient to establish as statistically significant correlations having success rates $\gtrsim 80 \%$, while a sample of 36 is large enough to establish correlations having success rates as low as $\sim 70 \%$. A sample larger than 50 is needed to show correlations having a $\sim 65 \%$ success rate to be statistically significant. 
Since different temporal windows may have different numbers of CME-productive active regions, the column sums are not necessarily constant among different windows. Since the same threshold rule is used for each window, the sums of the rows are constant. This allows the CME-prediction success rates for a measure to differ by as little as $\sim 3 \%$ between different temporal windows (e.g., the difference in the success rates in the center and right entries in Table 6). However, for any given measure and any given temporal window, the step size is $\sim 6 \%$ for a sample size of 36 , and $(2 / N) \times 100 \%$ for a sample size of $\mathrm{N}$.

Further, two contingency tables can have the same success rate yet different confidence levels. For a given success rate, the confidence level of the correlation is greater for a larger sample (larger number of trails). To illustrate the difference between confidence level and success rate, consider what occurs if the sample size is doubled by doubling each number in the middle $2 \times 2$ contingency table in Table 6 . The resulting confidence level will increase to $99.996 \%$, the success rate will remain $78 \%$, and the uncertainty (step size) of the success rate will drop to $3 \%[(2 / 72) \times 100 \%]$.

Figure 7 shows graphically how the probability $P$ for a correlation to occur by random chance changes as a function of sample size and success rate. Figure 7 shows that once a success rate becomes statistically significant, $P$ decreases rapidly as the sample size grows. So a small difference in success rate can correspond to very different probabilities (confidence levels). Figure 7 indicates that we will have to increase our sample size to 50 or more in order to show that a correlation with a $65 \%$ success rate, such as the correlation of active-region total magnetic flux with active-region CME productivity, is a statistically significant correlation.

Antiochos, S. K., Devore, C. R., \& Klimchuk, J. A. 1999, ApJ, 510, 485

Berger, T. E., \& Lites, B. W. 2003, Sol. Phys., 213, 213

Canfield, R. C., Hudson, H. S., \& McKenzie, D. E. 1999, Geophys. Res. Lett., 26,627

Everitt, B. S. 1977, The Analysis of Contingency Tables (New York: Chapman and Hall)

Falconer, D. A. 1997, Sol. Phys., 176, 123 2001, J. Geophys. Res., 106(A11), 25185

Falconer, D. A., Gary, G. A., Moore, R. L., \& Porter, J. G. 2000, ApJ, 528, 1004

Falconer, D. A., Moore, R. L., \& Gary, G. A. 2002a, ApJ, 569, 1016

2002b, in Multi-Wavelength Observations of Coronal Structure and Dynamics, ed. P. H. C. Martens \& D. P. Cauffman (Amsterdam: Pergamon), 303 2003, J. Geophys. Res., 108(A10), 1380

Fisher, G. H., Longcope, D. W., Metcalf, T. R., \& Pevtsov, A. A. 1998, ApJ, 508,885

Gary, G. A., \& Hagyard, M. J. 1990, Sol. Phys., 126, 21

Gary, G. A., Moore, R. L., Hagyard, M.J., \& Haisch, B. M. 1987, ApJ, 314, 782

Gosling, J. T. 1996, ARA\&A, 34, 35

Hagyard, M. J., N. P. Cumings, E. A. West, \& J. E. Smith, 1982, Sol. Phys., 80, 33

Jones, H. P., Duvall, T. L., Jr., Harvey, J. W., Mahaffey, C. T., Schwitters, J. D., \& Simmons, J. E. 1992, Sol. Phys., 139, 211

\section{EFERENCES}

Kahler, S. 1987, Rev. Geophys., 25, 663

Krimigis, S. M. 1992, in the Astronomy and Astrophysics Encyclopedia, ed.

S. P. Maran (New York: Van Nostrand Reinhold), 332

Leka, K. D., \& Barnes, G. 2003, ApJ, 595, 1296

Moore, R., \& Rabin, D. 1985, ARA\&A, 23, 239

Moore, R. L., \& Roumeliotis, G. 1992, Eruptive Solar Flares, ed. Z. Svestka, B. V. Jackson, \& M. E. Machado (Berlin: Springer), 69

Pevtsov, A. A., Canfield, R. C., \& Metcalf, T. R. 1995, ApJ, 440, L109

Phillips, A. D., MacNeice, P. J., \& Antiochos, S. K. 2005, ApJ, 624, L129

Reames, D. V. 1999, Space Sci. Rev., 90, 413

. 2001 in Space Weather, ed. P. Song, H. J. Singer, \& G. L. Siscoe (Geophys. Monogr. 125;Washington: AGU), 101

Scherrer, P. H., et al. 1995, Sol. Phys., 162, 129

Sturrock, P. A. 1989, Sol. Phys., 121, 387

Suess, S. T., \& Tsurutani, B. T., ed. 1998, From the Sun: Auroras, Magnetic Storms, Solar Flares, Cosmic Rays (Washington: AGU)

Wagner, W. J. 1984, ARA\&A, 22, 267

Webb, D. F., Krieger, A. S., \& Rust, D. M. 1976, Sol. Phys., 48, 159

West, E. A., Hagyard, M. J., Gary, G. A., Smith, J. E., Adams, M., \& Cloyd, R. A. 2002, Proc. SPIE, 4481, 270

Zirin, H. 1988, Astrophysics of the Sun (Cambridge: Cambridge Univ. Press) Zirin, H., \& Liggett, M. A. 1987, Sol. Phys., 113, 267 